\title{
Robustness of non-autonomous attractors for a family of nonlocal reaction-diffusion equations without uniqueness
}

\author{
Tomás Caraballo • Marta \\ Herrera-Cobos • Pedro Marín-Rubio
}

Received: date / Accepted: date

\begin{abstract}
In this paper, we consider a non-autonomous nonlocal reactiondiffusion equation with a small perturbation in the nonlocal diffusion term and the non-autonomous force. Under the assumptions imposed on the viscosity function, the uniqueness of weak solutions cannot be guaranteed. In this multi-valued framework, the existence of weak solutions and minimal pullback attractors in the $L^{2}$-norm are analysed. In addition, some relationships between the attractors of the universe of fixed bounded sets and those associated to a universe given by a tempered condition are established. Finally, the upper semicontinuity property of pullback attractors w.r.t. the parameter is proved. Indeed, under suitable assumptions, we prove that the family of pullback attractors converges to the corresponding global compact attractor associated to the autonomous nonlocal limit problem when the parameter goes to zero.
\end{abstract}

Keywords nonlocal diffusion - reaction-diffusion equations without uniqueness $\cdot$ pullback attractors · upper semicontinuity of attractors $\cdot$ multivalued dynamical systems.

Mathematics Subject Classification (2000) Primary 35B40 · Secondary 35B41, 35K57, 37L30

T. Caraballo

Departamento de Ecuaciones Diferenciales y Análisis Numérico, Universidad de Sevilla

Apdo. de Correos 1160, 41080 Sevilla, Spain

E-mail: caraball@us.es

M. Herrera-Cobos

Departamento de Ecuaciones Diferenciales y Análisis Numérico, Universidad de Sevilla Apdo. de Correos 1160, 41080 Sevilla, Spain

E-mail: mhc@us.es

P. Marín-Rubio

Departamento de Ecuaciones Diferenciales y Análisis Numérico, Universidad de Sevilla Apdo. de Correos 1160, 41080 Sevilla, Spain

E-mail: pmr@us.es 


\section{Introduction and statement of the problem}

Recently nonlocal problems have attracted attention of many authors because nonlocal terms allow to give more accurate results, for instance, in physics (cf. $[8,16,54])$, engineering (cf. $[39,6,37,40])$, and population dynamics (cf. [42,23, $21])$. If one considers the parabolic diffusion equation

$$
\frac{d u}{d t}-\Delta u=f
$$

being $u$ the density of population, we are not considering, for instance, what the behaviour of the population is when an area is overcrowded or isolated. However, if the diffusion term were nonlocal, this behaviour could be considered. Therefore, the obtained results would be more accurate if these effects were taken into account (e.g., cf. $[22,23]$ ). A variant of problem (1) in search of the higher accuracy in the results is settled in [42]. The nonlocal parabolic equation

$$
\frac{d u}{d t}-a\left(\int_{\Omega} u(x) d x\right) \Delta u=f
$$

is considered, where $a \in C\left(\mathbb{R} ; \mathbb{R}_{+}\right)$and there exists two positive constants $m, M$ such that

$$
0<m \leq a(\xi) \leq M \quad \forall \xi \in \mathbb{R} .
$$

These are natural conditions of non-degeneracy of $a$ in order to the solution exists not only in finite-time intervals (see [42] for more details). Observe that this equation is much more complex than (1), since, for instance, some common manipulations such as multiplying by $u_{t}$ do not give any information in the a priori estimates, unlike what happens in the local case (see [3, Chapter 2, p. 32] for more details). Assuming also that the function $a$ is globally Lipschitz, the uniqueness of a weak solution is guaranteed. Since then, Chipot and their collaborators have studied the asymptotic behaviour of the solution of nonlocal evolution problems with uniqueness similar to (2) considering mixed boundary conditions (cf. $[17,26]$ ), different nonlocal terms (cf. $[22,24,27]$ ), and even they have analysed other types of nonlocal evolution equations like the nonlocal plaplacian evolution equation (cf. [25]). To that end, different techniques have been applied such as dynamical systems (cf. [42,24,28]), energy functionals, global minimizers, and Lyapunov function (cf. $[27,25]$ ), which does not always exist (see [28] for more details). In addition, some results that establish order relationships among two stationary solutions and the long-time behaviour of the solution of the evolution problem have also been studied (cf. [23, 24,1719]).

In the context of attractors, there are several choices to study the asymptotic behaviour of the solutions of evolution problems. One can prove the existence of the global compact attractor in the autonomous framework as in $[42,1]$. However, when the equation possesses time-dependent terms, several approaches from non-autonomous dynamical systems can be used. Namely, one can do attempts with uniform attractors (cf. [20]), skew-product flows (cf. 
[50]), and pullback attractors (see [38] for more details; also related to random dynamical systems, cf. [30]). In this paper, we will choose this last approach because it allows us to minimize the assumptions on the forcing terms and the resultant objects are strictly invariant in a suitable sense. Indeed, we will not study the pullback attractors of "fixed" bounded sets, but for a class (called universe) of families which can move in time and fulfil a tempered condition (cf. $[11,9,33]$ ).

The existence of pullback attractors in $L^{2}(\Omega)$ for the following nonlocal non-autonomous equation

$$
\frac{d u}{d t}-a(l(u)) \Delta u=f(u)+h(t) \quad \text { in } \Omega \times(\tau, \infty)
$$

with homogeneous Dirichlet boundary conditions was studied in [12] when $f$ was a sublinear function (see also [29] for a particular close result with exponential decay to zero). Later, we have proved an analogous result (submitted for publication) for $f$ satisfying

$$
-\kappa-\alpha_{1}|s|^{p} \leq f(s) s \leq \kappa-\alpha_{2}|s|^{p} \quad \forall s \in \mathbb{R},
$$

where $\alpha_{1}, \alpha_{2}$, and $\kappa$ are positive constants and $p \geq 2$ (see also [51] for a particular case). In both papers, the function $a$ is assumed to be locally Lipchitz and the function $f$ fulfilled a monotonicity condition. These two assumptions lead to the uniqueness of a weak solution. In this paper, we get rid of them to prove the existence of minimal pullback attractors in the $L^{2}$-norm for the dynamical system associated to the following perturbed non-autonomous nonlocal reaction-diffusion problem

$$
\left(P_{\varepsilon}\right)\left\{\begin{array}{l}
\frac{d u}{d t}-(1-\varepsilon) a(l(u)) \Delta u=f(u)+\varepsilon h(t) \text { in } \Omega \times(\tau, \infty), \\
u=0 \text { on } \partial \Omega \times(\tau, \infty), \\
u(x, \tau)=u_{\tau}(x) \text { in } \Omega,
\end{array}\right.
$$

where $\Omega \subset \mathbb{R}^{N}$ is a nonempty bounded open set, $\tau \in \mathbb{R}, \varepsilon \in[0,1), f \in C(\mathbb{R})$ fulfils (4), and $l \in\left(L^{2}(\Omega)\right)^{\prime}$.

Observe that from (4), we can deduce that there exists $\beta>0$ such that

$$
|f(s)| \leq \beta\left(|s|^{p-1}+1\right) \quad \forall s \in \mathbb{R} .
$$

Under the more relaxed assumption of less regularity on the function $a$ and due to the possible lack of uniqueness, the existence of minimal pullback attractors in $L^{2}(\Omega)$ is carried out using the theory of non-autonomous multi-valued dynamical systems (see $[47,43,13,36,46,2,3]$ for more details). Moreover, relationships between these attractors and the attractors of the universe of fixed bounded sets of $L^{2}(\Omega)$ will be also analysed in this paper making use of the techniques in [46].

In addition, we will be interested in studying the upper semicontinuity of attractors with respect to the parameter $\varepsilon$ (cf. [15, Chapter 3]). Many authors have been interested in studying this robustness property in different 
frameworks. For instance, in the random context, it is studied by Caraballo, Langa, and Robinson in [10]. There, the upper semicontinuity w.r.t. a parameter is proved for two problems, a reaction-diffusion and Navier-Stokes equations, both with a small random perturbation involving additive noise. In [14], Carvalho, Rodrigues, and Dłotko prove that diffusively coupled abstract semilinear parabolic systems synchronize by proving the upper semicontinuity of the associated attractors and the one of a limiting problem. Later, Arrieta, Carvalho, and Rodríguez-Bernal prove in [7] this property for a nonlinear second-order parabolic equation for which the diffusion coefficient was large in a subdomain of $\Omega$. In a multi-valued framework, in [43] the upper semicontinuous behaviour of a family of attractors corresponding to a general class of parameterized delay differential equations posed in potentially different state spaces is studied. Now, this property will be analysed in this paper in the nonlocal framework. As far as we know, in the previous literature there are no results on the existence of attractors in this multi-valued framework for problems $\left(P_{\varepsilon}\right)$ neither on their upper semicontinuous behaviour.

The structure of the paper is as follows. The existence of weak solutions is analysed in Section 2 by using the Faedo-Galerkin approximations and compactness arguments (cf. $[41,48]$ ). Section 3 is devoted to recall some abstract results on the theory of multi-valued non-autonomous dynamical systems. These results are used in Section 4 to prove the existence of minimal pullback attractors in $L^{2}(\Omega)$. To that end, we apply an energy method which relies on the continuity of weak solutions (see $[34,35,44,46,33])$. Thereupon, we establish some relationships between pullback attractors. Finally, under some additional assumptions, we obtain the upper semicontinuity of these pullback attractors as $\varepsilon \rightarrow 0$ in Section 5 . Indeed, we prove that the family of pullback attractors indexed by $\varepsilon$ converges to the attractor associated to $\left(P_{0}\right)$ when $\varepsilon \rightarrow 0$. [Of course, here after $\left(P_{0}\right)$ denotes the problem $\left(P_{\varepsilon}\right)$ with $\varepsilon=0$.]

Before to start, let us introduce some notation that will be used in the sequel. As usual, the inner product in $L^{2}(\Omega)$ will be denoted by $(\cdot, \cdot)$ and by | - | its associated norm. The inner product in $H_{0}^{1}(\Omega)$ given by the product in $\left(L^{2}(\Omega)\right)^{N}$ of the gradients will be represented by $((\cdot, \cdot))$ and by $\|\cdot\|$ its associated norm. By $\langle\cdot, \cdot\rangle$ we represent the duality product between $H^{-1}(\Omega)$ and $H_{0}^{1}(\Omega)$ and by $\|\cdot\|_{*}$ the norm in $H^{-1}(\Omega)$. We identify $L^{2}(\Omega)$ with its dual, and therefore we have the chain of compact and dense embeddings $H_{0}^{1}(\Omega) \subset$ $L^{2}(\Omega) \subset H^{-1}(\Omega)$. Observe that as a result of the previous identification, we can make an abuse of the notation considering $l \in L^{2}(\Omega)$ and denoting $(l, u)$ like $l(u)$. The duality product between $L^{p}(\Omega)$ and $L^{q}(\Omega)$ (where $q$ is the conjugate exponent of $p$ ) will be denoted by $(\cdot, \cdot)$ and the norm in $L^{p}(\Omega)$ will be represented by $\|\cdot\|_{L^{p}(\Omega)}$. We also denote by $\langle\cdot, \cdot\rangle$ the duality product between $H^{-1}(\Omega)+L^{q}(\Omega)$ and $H_{0}^{1}(\Omega) \cap L^{p}(\Omega)$. Finally, the norm in $L^{r}(\tau, T ; X)$, where $r \geq 1$ and $X$ is a separable Banach space, will be denoted by $\|\cdot\|_{L^{r}(\tau, T ; X)}$.

In what follows we assume that $h \in L_{\text {loc }}^{2}\left(\mathbb{R} ; H^{-1}(\Omega)\right)$ and $u_{\tau} \in L^{2}(\Omega)$. 
Definition $1 \mathrm{~A}$ weak solution to $\left(P_{\varepsilon}\right)$ is a function $u$ that, for all $T>\tau$, belongs to $L^{\infty}\left(\tau, T ; L^{2}(\Omega)\right) \cap L^{2}\left(\tau, T ; H_{0}^{1}(\Omega)\right) \cap L^{p}\left(\tau, T ; L^{p}(\Omega)\right)$, with $u(\tau)=$ $u_{\tau}$, and such that for all $v \in H_{0}^{1}(\Omega) \cap L^{p}(\Omega)$

$$
\frac{d}{d t}(u(t), v)+(1-\varepsilon) a(l(u(t)))((u(t), v))=(f(u(t)), v)+\varepsilon\langle h(t), v\rangle,
$$

where the previous equation must be understood in the sense of $\mathcal{D}^{\prime}(\tau, \infty)$.

Remark 1 If $u$ is a weak solution to $\left(P_{\varepsilon}\right)$, then, from (3), (5), and (6) we deduce that $u^{\prime} \in L^{2}\left(\tau, T ; H^{-1}(\Omega)\right)+L^{q}\left(\tau, T ; L^{q}(\Omega)\right)$ for any $T>\tau$. Therefore, $u \in C\left([\tau, \infty) ; L^{2}(\Omega)\right.$ ) (cf. [41, Théorème 2, p. 575]) and the initial datum in $\left(P_{\varepsilon}\right)$ makes sense. Moreover, the following energy equality holds

$$
\begin{aligned}
& |u(t)|^{2}+2(1-\varepsilon) \int_{s}^{t} a(l(u(r)))\|u(r)\|^{2} d r \\
= & |u(s)|^{2}+2 \int_{s}^{t}(f(u(r)), u(r)) d r+2 \varepsilon \int_{s}^{t}\langle h(r), u(r)\rangle d r
\end{aligned}
$$

for all $\tau \leq s \leq t$ (cf. [31, Théorème 2, p. 575], [53, Lemma 3.2, p. 71]).

\section{Existence of a weak solution}

In this section, we will study the existence of weak solution to $\left(P_{\varepsilon}\right)$. It is worth noting that we do not impose any assumptions of smoothness on $\Omega$.

Theorem 1 Assume that the function $a \in C\left(\mathbb{R} ; \mathbb{R}_{+}\right)$fulfils (3), $\varepsilon \in[0,1)$, $f \in C(\mathbb{R})$ satisfies (4), $h \in L_{\text {loc }}^{2}\left(\mathbb{R} ; H^{-1}(\Omega)\right)$, and $l \in L^{2}(\Omega)$. Then, for all $\tau \in \mathbb{R}, u_{\tau} \in L^{2}(\Omega)$, there exists at least one weak solution $u$ to $\left(P_{\varepsilon}\right)$.

Proof Consider fixed $T>\tau$. For each integer $n \geq 1$, we denote by

$$
u_{n}(t)=\sum_{j=1}^{n} \varphi_{n j}(t) w_{j}
$$

a local solution of

$$
\begin{cases}\frac{d}{d t}\left(u_{n}(t), w_{j}\right)+(1-\varepsilon) a\left(l\left(u_{n}(t)\right)\right)\left(\left(u_{n}(t), w_{j}\right)\right) & \\ =\left(f\left(u_{n}(t)\right), w_{j}\right)+\varepsilon\left\langle h(t), w_{j}\right\rangle, & \text { a.e. } t>\tau, \\ \left(u_{n}(\tau), w_{j}\right)=\left(u_{\tau}, w_{j}\right), & j=1, \ldots, n,\end{cases}
$$

where $\left\{w_{j}: j \geq 1\right\} \subset H_{0}^{1}(\Omega) \cap L^{p}(\Omega)$ is a Hilbert basis of $L^{2}(\Omega)$ such that $\bigcup_{n \in \mathbb{N}} \operatorname{span}\left\{w_{1}, \ldots, w_{n}\right\}$ is dense in $H_{0}^{1}(\Omega) \cap L^{p}(\Omega)$. Now, multiplying by $\varphi_{n j}(t)$ in (8), summing from $j=1$ to $n$, and using (3), we obtain

$$
\frac{1}{2} \frac{d}{d t}\left|u_{n}(t)\right|^{2}+(1-\varepsilon) m\left\|u_{n}(t)\right\|^{2} \leq\left(f\left(u_{n}(t)\right), u_{n}(t)\right)+\varepsilon\left\langle h(t), u_{n}(t)\right\rangle
$$


a.e. $t \in\left(\tau, T_{n}\right)$, where $\left(\tau, T_{n}\right)$ is an interval of existence of solutions to (8) by the Carathéodory Theorem.

From (4),

$$
\left(f\left(u_{n}(t)\right), u_{n}(t)\right) \leq \kappa|\Omega|-\alpha_{2}\left\|u_{n}(t)\right\|_{L^{p}(\Omega)}^{p} \quad \text { a.e. } t \in\left(\tau, T_{n}\right) .
$$

On the other hand, using the Cauchy inequality (cf. [32, Appendix B, p. $622]$ ) and taking into account that $\varepsilon \in[0,1)$, we have

$$
\left\langle h(t), u_{n}(t)\right\rangle \leq \frac{1}{2(1-\varepsilon) m}\|h(t)\|_{*}^{2}+\frac{(1-\varepsilon) m}{2}\left\|u_{n}(t)\right\|^{2} \quad \text { a.e. } t \in\left(\tau, T_{n}\right) .
$$

Therefore, taking these two inequalities into account, from (9) we deduce

$\frac{d}{d t}\left|u_{n}(t)\right|^{2}+(1-\varepsilon) m\left\|u_{n}(t)\right\|^{2}+2 \alpha_{2}\left\|u_{n}(t)\right\|_{L^{p}(\Omega)}^{p} \leq 2 \kappa|\Omega|+\frac{\varepsilon}{(1-\varepsilon) m}\|h(t)\|_{*}^{2}$

a.e. $t \in\left(\tau, T_{n}\right)$. Now, integrating between $\tau$ and $t \in\left(\tau, T_{n}\right)$,

$$
\begin{aligned}
& \left|u_{n}(t)\right|^{2}+(1-\varepsilon) m \int_{\tau}^{t}\left\|u_{n}(s)\right\|^{2} d s+2 \alpha_{2} \int_{\tau}^{t}\left\|u_{n}(s)\right\|_{L^{p}(\Omega)}^{p} d s \\
\leq & \left|u_{\tau}\right|^{2}+2 \kappa \Omega(T-\tau)+\frac{\varepsilon}{(1-\varepsilon) m} \int_{\tau}^{T}\|h(s)\|_{*}^{2} d s .
\end{aligned}
$$

From the above a priori estimate, we deduce that solutions to (8) are defined in the whole interval $[\tau, T]$, and the sequence $\left\{u_{n}\right\}$ is bounded in $L^{\infty}\left(\tau, T ; L^{2}(\Omega)\right) \cap$ $L^{2}\left(\tau, T ; H_{0}^{1}(\Omega)\right) \cap L^{p}\left(\tau, T ; L^{p}(\Omega)\right)$.

Now, using (5) and the boundedness of $\left\{u_{n}\right\}$ in $L^{p}\left(\tau, T ; L^{p}(\Omega)\right)$, we deduce that $\left\{f\left(u_{n}\right)\right\}$ is bounded in $L^{q}\left(\tau, T ; L^{q}(\Omega)\right)$.

The boundedness of $\left\{-a\left(l\left(u_{n}\right)\right) \Delta u_{n}\right\}$ is obtained making use of the boundedness of $\left\{u_{n}\right\}$ in $L^{2}\left(\tau, T ; H_{0}^{1}(\Omega)\right)$ and $(3)$.

Thus, we deduce that there exist $u \in L^{\infty}\left(\tau, T ; L^{2}(\Omega)\right) \cap L^{2}\left(\tau, T ; H_{0}^{1}(\Omega)\right) \cap$ $L^{p}\left(\tau, T ; L^{p}(\Omega)\right), \xi_{1} \in L^{q}\left(\tau, T ; L^{q}(\Omega)\right)$ and $\xi_{2} \in L^{2}\left(\tau, T ; H^{-1}(\Omega)\right)$, and a subsequence of $\left\{u_{n}\right\}$ (relabeled the same) such that

$$
\left\{\begin{aligned}
u_{n} \stackrel{*}{\rightarrow} u & \text { weakly-star in } L^{\infty}\left(\tau, T ; L^{2}(\Omega)\right), \\
u_{n} \rightarrow u & \text { weakly in } L^{2}\left(\tau, T ; H_{0}^{1}(\Omega)\right), \\
u_{n} \rightarrow u & \text { weakly in } L^{p}\left(\tau, T ; L^{p}(\Omega)\right), \\
f\left(u_{n}\right) \rightarrow \xi_{1} & \text { weakly in } L^{q}\left(\tau, T ; L^{q}(\Omega)\right), \\
-a\left(l\left(u_{n}\right)\right) \Delta u_{n} \rightarrow \xi_{2} & \text { weakly in } L^{2}\left(\tau, T ; H^{-1}(\Omega)\right) .
\end{aligned}\right.
$$

To prove that $\xi_{1}=f(u)$ and $\xi_{2}=-a(l(u)) \Delta u$, we will argue analogously as in [49, Lemma 2.1] or [4, Proposition 4.1]. Consider fixed $w \in \operatorname{span}\left[w_{1}, \ldots, w_{n}\right]$. 
Integrating in (8) between $t$ and $t+b$, with $b \in(0, T-\tau), t \in(\tau, T-b)$, and using (3) and the Hölder inequality, we have

$$
\begin{aligned}
& \left(u_{n}(t+b)-u_{n}(t), w\right) \\
\leq & (1-\varepsilon) M \int_{t}^{t+b}\left\|u_{n}(s)\right\|\|w\| d s+\int_{t}^{t+b}\left\|f\left(u_{n}(s)\right)\right\|_{L^{q}(\Omega)}\|w\|_{L^{p}(\Omega)} d s \\
& +\varepsilon \int_{t}^{t+b}\|h(s)\| *\|w\| d s \\
\leq & (1-\varepsilon) b^{1 / 2} M\|w\|\left\|u_{n}\right\|_{L^{2}\left(\tau, T ; H_{0}^{1}(\Omega)\right)}+b^{1 / p}\|w\|_{L^{p}(\Omega)}\left\|f\left(u_{n}\right)\right\|_{L^{q}\left(\tau, T ; L^{q}(\Omega)\right)} \\
& +b^{1 / 2} \varepsilon\|w\|\|h\|_{L^{2}\left(\tau, T ; H^{-1}(\Omega)\right)} .
\end{aligned}
$$

Since $\left\{u_{n}\right\}$ and $\left\{f\left(u_{n}\right)\right\}$ are bounded in $L^{2}\left(\tau, T ; H_{0}^{1}(\Omega)\right)$ and $L^{q}\left(\tau, T ; L^{q}(\Omega)\right)$ respectively, there exists a positive constant $C_{\varepsilon}$ such that

$$
\left(u_{n}(t+b)-u_{n}(t), w\right) \leq C_{\varepsilon}\left(b^{1 / 2}+b^{1 / p}\right)\left(\|w\|+\|w\|_{L^{p}(\Omega)}\right) .
$$

Then, it fulfils a.e. $t \in(\tau, T-b)$

$\left|u_{n}(t+b)-u_{n}(t)\right|^{2} \leq C_{\varepsilon}\left(b^{1 / 2}+b^{1 / p}\right)\left(\left\|u_{n}(t+b)-u_{n}(t)\right\|+\left\|u_{n}(t+b)-u_{n}(t)\right\|_{L^{p}(\Omega)}\right)$.

Now, integrating between $\tau$ and $T-b$, we have

$\int_{\tau}^{T-b}\left|u_{n}(t+b)-u_{n}(t)\right|^{2} d t \leq 2 C_{\varepsilon}\left(b^{1 / 2}+b^{1 / p}\right) \int_{\tau}^{T}\left(\left\|u_{n}(r)\right\|+\left\|u_{n}(r)\right\|_{L^{p}(\Omega)}\right) d r$.

Thereupon, using the Hölder inequality,

$$
\begin{aligned}
& \int_{\tau}^{T-b}\left|u_{n}(t+b)-u_{n}(t)\right|^{2} d t \\
& \leq 2 C_{\varepsilon}\left(b^{1 / 2}+b^{1 / p}\right)\left((T-\tau)^{1 / 2}\left\|u_{n}\right\|_{L^{2}\left(\tau, T ; H_{0}^{1}(\Omega)\right)}+(T-\tau)^{1 / q}\left\|u_{n}\right\|_{L^{p}\left(\tau, T ; L^{p}(\Omega)\right)}\right) .
\end{aligned}
$$

As a result of the previous estimates, there exists a positive constant $\widehat{C}_{\varepsilon}(T)$ such that

$$
\int_{\tau}^{T-b}\left|u_{n}(t+b)-u_{n}(t)\right|^{2} d t \leq \widehat{C}_{\varepsilon}(T)\left(b^{1 / 2}+b^{1 / p}\right) \quad \forall n \geq 1 \quad \forall b \in(0, T-\tau) .
$$

Therefore,

$$
\lim _{b \rightarrow 0} \sup _{n} \int_{\tau}^{T-b}\left|u_{n}(t+b)-u_{n}(t)\right|^{2} d t=0 .
$$

In addition, taking into account that $\left\{u_{n}\right\}$ is bounded in $L^{\infty}\left(\tau, T ; L^{2}(\Omega)\right)$, it satisfies

$$
\lim _{b \rightarrow 0} \sup _{n}\left(\int_{\tau}^{\tau+b}\left|u_{n}(t)\right|^{2} d t+\int_{T-b}^{T}\left|u_{n}(t)\right|^{2} d t\right)=0
$$


Then, since the embedding $H_{0}^{1}(\Omega) \hookrightarrow L^{2}(\Omega)$ is compact and taking into account (11) and (12), applying [52, Theorem 13.2, p. 97] and [52, Remark 13.1, p.100], we have that the sequence $\left\{u_{n}\right\}$ is relatively compact in $L^{2}\left(\tau, T ; L^{2}(\Omega)\right)$. From this, applying [41, Lemme 1.3 , p. 12], we identify $\xi_{1}$ and $\xi_{2}$ in (10), namely

$$
\begin{aligned}
f\left(u_{n}\right) & \rightarrow f(u) \quad \text { weakly in } L^{q}\left(\tau, T ; L^{q}(\Omega)\right) \\
a\left(l\left(u_{n}\right)\right) u_{n} & \rightarrow a(l(u)) u \quad \text { weakly in } L^{2}\left(\tau, T ; H_{0}^{1}(\Omega)\right) .
\end{aligned}
$$

Then, if we consider fixed $n, \varphi \in \mathcal{D}(\tau, T)$, and $w \in \operatorname{span}\left[w_{1}, \ldots, w_{n}\right]$, it holds for all $\mu>n$

$$
\begin{aligned}
& -\int_{\tau}^{T}\left(u_{\mu}(t), w\right) \varphi^{\prime}(t) d t+(1-\varepsilon) \int_{\tau}^{T} a\left(l\left(u_{\mu}(t)\right)\right)\left\langle-\Delta u_{\mu}(t), w\right\rangle \varphi(t) d t \\
= & \int_{\tau}^{T}\left(f\left(u_{\mu}(t)\right), w\right) \varphi(t) d t+\varepsilon \int_{\tau}^{T}\langle h(t), w\rangle \varphi(t) d t .
\end{aligned}
$$

Now, making $\mu \rightarrow \infty$, from (10), (13), and (14), we obtain

$$
\begin{aligned}
& -\int_{\tau}^{T}(u(t), w) \varphi^{\prime}(t) d t+(1-\varepsilon) \int_{\tau}^{T} a(l(u(t)))\langle-\Delta u(t), w\rangle \varphi(t) d t \\
= & \int_{\tau}^{T}(f(u(t)), w) \varphi(t) d t+\varepsilon \int_{\tau}^{T}\langle h(t), w\rangle \varphi(t) d t,
\end{aligned}
$$

for all $w \in H_{0}^{1}(\Omega) \cap L^{p}(\Omega)$, since $\bigcup_{n \in \mathbb{N}} \operatorname{span}\left\{w_{1}, \ldots, w_{n}\right\}$ is dense in $H_{0}^{1}(\Omega) \cap$ $L^{p}(\Omega)$.

Therefore,

$$
\frac{d u}{d t}-(1-\varepsilon) a(l(u)) \Delta u=f(u)+\varepsilon h \quad \text { in } \mathcal{D}^{\prime}\left(\tau, T ; H^{-1}(\Omega)+L^{q}(\Omega)\right),
$$

and taking into account the regularity of $f(u),-a(l(u)) \Delta u$, and $h$, it fulfils that $u^{\prime}$ belongs to $L^{2}\left(\tau, T ; H^{-1}(\Omega)\right)+L^{q}\left(\tau, T ; L^{q}(\Omega)\right)$.

Thus, by the regularity of $u$ and $u^{\prime}$, it holds that $u \in C\left([\tau, T] ; L^{2}(\Omega)\right)$. Finally, we only need to check that $u(\tau)=u_{\tau}$.

On the one hand, we fix $n \geq 1, \varphi \in H^{1}(\tau, T)$ such that $\varphi(T)=0$ and $\varphi(\tau) \neq 0$, and $w \in \operatorname{span}\left[w_{1}, \ldots, w_{n}\right]$, and consider $\mu>n$. Then, at light of (8) we have

$$
\begin{aligned}
& -\left(u_{\tau}, w\right) \varphi(\tau)-\int_{\tau}^{T}\left(u_{\mu}(t), w\right) \varphi^{\prime}(t) d t+(1-\varepsilon) \int_{\tau}^{T} a\left(l\left(u_{\mu}(t)\right)\right)\left\langle-\Delta u_{\mu}(t), w\right\rangle \varphi(t) d t \\
= & \int_{\tau}^{T}\left(f\left(u_{\mu}(t)\right), w\right) \varphi(t) d t+\varepsilon \int_{\tau}^{T}\langle h(t), w\rangle \varphi(t) d t .
\end{aligned}
$$

Now, taking limit when $\mu \rightarrow \infty$,

$$
\begin{aligned}
& -\left(u_{\tau}, w\right) \varphi(\tau)-\int_{\tau}^{T}(u(t), w) \varphi^{\prime}(t) d t+(1-\varepsilon) \int_{\tau}^{T} a(l(u(t)))\langle-\Delta u(t), w\rangle \varphi(t) d t \\
= & \int_{\tau}^{T}(f(u(t)), w) \varphi(t) d t+\varepsilon \int_{\tau}^{T}\langle h(t), w\rangle \varphi(t) d t .
\end{aligned}
$$


On the other hand, from (6) we deduce

$$
\begin{aligned}
& -(u(\tau), w) \varphi(\tau)-\int_{\tau}^{T}(u(t), w) \varphi^{\prime}(t) d t+(1-\varepsilon) \int_{\tau}^{T} a(l(u(t)))\langle-\Delta u(t), w\rangle \varphi(t) d t \\
= & \int_{\tau}^{T}(f(u(t)), w) \varphi(t) d t+\varepsilon \int_{\tau}^{T}\langle h(t), w\rangle \varphi(t) d t .
\end{aligned}
$$

Then, comparing (15) with this last expression, it holds that $\left(u_{\tau}, w\right) \varphi(\tau)=$ $(u(\tau), w) \varphi(\tau)$ with $w \in \operatorname{span}\left[w_{1}, \ldots, w_{n}\right]$. Finally, taking into account that $\varphi(\tau) \neq 0$ and $\left\{w_{j}\right\}$ is a Hilbert basis of $L^{2}(\Omega)$, we conclude that $u(\tau)=u_{\tau}$.

Since a weak solution on an arbitrary finite time-interval $[\tau, T]$ has been obtained, then we may now repeat the argument, say, on an interval of the form $[T, T+1]$, then on $[T+1, T+2]$, etcetera, concatenating these solutions to obtain a weak solution well-defined globally in time.

\section{Abstract results on multi-valued process and pullback attractors}

In this section, we briefly recall some abstract results on multi-valued nonautonomous dynamical systems (cf. $[47,43,13,36,46,2,3]$ ) which we will use in the following section to prove the main results of this paper. In addition, we will show results which allow us to establish relationships between pullback attractors (cf. [46]).

Let $\left(X, d_{X}\right)$ be a metric space and $\mathbb{R}_{d}^{2}=\left\{(t, s) \in \mathbb{R}^{2}: t \geq s\right\}$. In what follows, we denote by $\mathcal{P}(X)$ the family of all nonempty subsets of $X$.

Definition 2 A multi-valued process (also called multi-valued non-autonomous dynamical system) $U$ on $X$ is a family of mappings $U(t, \tau): X \mapsto \mathcal{P}(X)$ for any pair $(t, \tau) \in \mathbb{R}_{d}^{2}$, such that

(i) $U(\tau, \tau) x=\{x\} \forall \tau \in \mathbb{R} \forall x \in X$.

(ii) $U(t, \tau) x \subset U(t, s)(U(s, \tau) x) \forall \tau \leq s \leq t \forall x \in X$, where $U(t, \tau) W:=$ $\bigcup_{y \in W} U(t, \tau) y \forall W \subset X$.

Observe that if the relationship given in (ii) is an equality instead of an inclusion, the multi-valued process $U$ is called strict.

Definition 3 A multi-valued process $U$ on $X$ is upper-semicontinuous if the mapping $U(t, \tau)$ is upper-semicontinuous from $X$ into $\mathcal{P}(X)$ for all $(t, \tau) \in \mathbb{R}_{d}^{2}$, i.e. for any $x \in X$ and for every neighborhood $\mathcal{N}$ in $X$ of the set $U(t, \tau) x$, there exists a value $\varepsilon>0$ such that $U(t, \tau) y \subset \mathcal{N}$ provided that $d_{X}(x, y)<\varepsilon$.

Now, we consider a universe $\mathcal{D}$, that is a nonempty class of families parameterized in time $\widehat{D}=\{D(t): t \in \mathbb{R}\} \subset \mathcal{P}(X)$ and a family of nonempty sets $\widehat{D}_{0}=\left\{D_{0}(t): t \in \mathbb{R}\right\} \subset \mathcal{P}(X)$.

Definition 4 A universe $\mathcal{D}$ is inclusion-closed if given $\widehat{D} \in \mathcal{D}$ and $\widehat{D}^{\prime}=\left\{D^{\prime}(t)\right.$ : $t \in \mathbb{R}\} \subset \mathcal{P}(X)$ with $D^{\prime}(t) \subset D(t)$ for all $t \in \mathbb{R}$, it fulfils that $\widehat{D}^{\prime} \in \mathcal{D}$. 
Definition 5 A family $\widehat{D}_{0}=\left\{D_{0}(t): t \in \mathbb{R}\right\}$ is said to be pullback $\mathcal{D}$ absorbing for a multi-valued process $U$ if for every $t \in \mathbb{R}$ and $\widehat{D} \in \mathcal{D}$, there exists $\tau(\widehat{D}, t) \leq t$ such that

$$
U(t, \tau) D(\tau) \subset D_{0}(t) \quad \forall \tau \leq \tau(\widehat{D}, t)
$$

Definition 6 Given a family of nonempty sets $\widehat{D}=\{D(t): t \in \mathbb{R}\} \subset \mathcal{P}(X)$, a multi-valued process $U$ on $X$ is pullback $\widehat{D}$-asymptotically compact if for any $t \in \mathbb{R}$ and any sequences $\left\{\tau_{n}\right\} \subset(-\infty, t]$ and $\left\{x_{n}\right\} \subset X$ such that $\tau_{n} \rightarrow-\infty$ and $x_{n} \in D\left(\tau_{n}\right)$, it fulfils that any sequence $\left\{y_{n}\right\}$ is relatively compact in $X$, where $y_{n} \in U\left(t, \tau_{n}\right) x_{n}$ for all $n$.

Definition 7 A multi-valued process $U$ on $X$ is pullback $\mathcal{D}$-asymptotically compact if it is pullback $\widehat{D}$-asymptotically compact for any $\widehat{D} \in \mathcal{D}$.

Definition 8 A pullback $\mathcal{D}$-attractor for a multi-valued process $U$ on $X$ is a family $\mathcal{A}_{\mathcal{D}}=\left\{\mathcal{A}_{\mathcal{D}}(t): t \in \mathbb{R}\right\} \subset \mathcal{P}(X)$ such that

1. for any $t \in \mathbb{R}$, the set $\mathcal{A}_{\mathcal{D}}(t)$ is a nonempty compact subset of $X$;

2. $\mathcal{A}_{\mathcal{D}}$ is pullback $\mathcal{D}$-attracting, i.e.

$$
\lim _{\tau \rightarrow-\infty} \operatorname{dist}_{X}\left(U(t, \tau) D(\tau), \mathcal{A}_{\mathcal{D}}(t)\right)=0 \quad \forall \widehat{D} \in \mathcal{D} \quad \forall t \in \mathbb{R},
$$

where $\operatorname{dist}_{X}(\cdot, \cdot)$ denotes the Hausdorff semi-distance in $X$ between two subsets of $X$;

3. $\mathcal{A}_{\mathcal{D}}$ is negatively invariant, i.e.

$$
\mathcal{A}_{\mathcal{D}}(t) \subset U(t, \tau) \mathcal{A}_{\mathcal{D}}(\tau) \quad \forall(t, \tau) \in \mathbb{R}_{d}^{2}
$$

A pullback $\mathcal{D}$-attractor $\mathcal{A}_{\mathcal{D}}$ is said to be minimal if it satisfies that if there exists another family of closed sets $\widehat{C}=\{C(t): t \in \mathbb{R}\}$ such that it is pullback $\mathcal{D}$-attracting, then $\mathcal{A}_{\mathcal{D}}(t) \subset C(t)$ for all $t \in \mathbb{R}$.

Observe that pullback attractors are not unique in general (cf. [45]); however, the minimal pullback attractor is, therefore, in the sense of minimality, one recovers uniqueness of pullback attractor.

In what follows, we denote the omega-limit set of $\widehat{D}_{0}$ at time $t$ by

$$
\Lambda\left(\widehat{D}_{0}, t\right)=\bigcap_{s \leq t}{\widehat{\bigcup} \underset{\tau \leq s}{ } U(t, \tau) D_{0}(\tau)}^{X}
$$

where $\overline{\{\ldots\}}^{X}$ denotes the closure in $X$.

The following result ensures the existence of a pullback $\mathcal{D}$-attractor for a multi-valued process $U$ (this generalizes slightly the results from $[13,3]$, see also [46]; the proof is very similar so we omit it). 
Theorem 2 Assume that $U$ is an upper-semicontinuous multi-valued process with closed values, $\widehat{D}_{0}=\left\{D_{0}(t): t \in \mathbb{R}\right\} \subset \mathcal{P}(X)$ is a pullback $\mathcal{D}$-absorbing family and also suppose that $U$ on $X$ is pullback $\widehat{D}_{0}$-asymptotically compact. Then, the family $\mathcal{A}_{\mathcal{D}}=\left\{\mathcal{A}_{\mathcal{D}}(t): t \in \mathbb{R}\right\}$ defined by

$$
\mathcal{A}_{\mathcal{D}}(t)=\varlimsup_{\widehat{D} \in \mathcal{D}} \Lambda(\widehat{D}, t) \quad \forall t \in \mathbb{R}
$$

is the minimal pullback $\mathcal{D}$-attractor and $\mathcal{A}_{\mathcal{D}}(t) \subset{\overline{D_{0}(t)}}^{X}$ for all $t \in \mathbb{R}$.

If $\mathcal{A}_{\mathcal{D}} \in \mathcal{D}$, then it is the unique family of $\mathcal{D}$ that satisfies the properties 1-3 given above. In addition, if the multi-valued process $U$ is strict, then $\mathcal{A}_{\mathcal{D}}$ is strictly invariant under the process $U$, i.e.

$$
\mathcal{A}_{\mathcal{D}}(t)=U(t, \tau) \mathcal{A}_{\mathcal{D}}(\tau) \quad \forall(t, \tau) \in \mathbb{R}_{d}^{2}
$$

We denote by $\mathcal{D}_{F}^{X}$ the universe of fixed nonempty bounded subsets of $X$, i.e. the class of all families $\widehat{D}$ of the form $\widehat{D}=\{D(t)=B: t \in \mathbb{R}\}$, where $B$ is a fixed nonempty bounded subset of $X$.

Now, we establish some relationships between pullback attractors (for more details see [46]).

Corollary 1 Under the assumptions of Theorem 2, if $\mathcal{D}_{F}^{X} \subset \mathcal{D}$, then $\mathcal{A}_{\mathcal{D}_{F}^{X}}=$ $\left\{\mathcal{A}_{\mathcal{D}_{F}^{X}}(t): t \in \mathbb{R}\right\}$, where

$$
\mathcal{A}_{\mathcal{D}_{F}^{X}}(t)=\varlimsup_{B \text { bounded }}^{\bigcup} \Lambda(B, t) \text {, }
$$

is the minimal pullback $\mathcal{D}_{F}^{X}$-attractor for the multi-valued process $U$ and the following relationship holds

$$
\mathcal{A}_{\mathcal{D}_{F}^{X}}(t) \subset \mathcal{A}_{D}(t) \quad \forall t \in \mathbb{R} .
$$

Corollary 2 Under the assumptions of the previous corollary, if there exists $T \in \mathbb{R}$ such that the set $\bigcup_{t \leq T} D_{0}(t)$ is bounded in $X$, then

$$
\mathcal{A}_{\mathcal{D}_{F}^{X}}(t)=\mathcal{A}_{\mathcal{D}}(t) \quad \forall t \leq T .
$$

\section{Minimal pullback attractors in $L^{2}(\Omega)$}

In this section, the existence of minimal pullback attractors in $L^{2}(\Omega)$ will be analysed using the results given in Section 3. In addition, we establish relationships between pullback attractors. Finally, under some additional assumptions, we will prove the upper semicontinuity of pullback attractors as $\varepsilon \rightarrow 0$.

In what follows, we denote by $\Phi^{\varepsilon}\left(\tau, u_{\tau}\right)$ the set of weak solutions to $\left(P_{\varepsilon}\right)$ in $[\tau, \infty)$ with initial datum $u_{\tau} \in L^{2}(\Omega)$. 
Now, we define a multi-valued map $U^{\varepsilon}: \mathbb{R}_{d}^{2} \times L^{2}(\Omega) \rightarrow \mathcal{P}\left(L^{2}(\Omega)\right)$ as

$$
U^{\varepsilon}(t, \tau) u_{\tau}=\left\{u(t): u \in \Phi^{\varepsilon}\left(\tau, u_{\tau}\right)\right\}, \quad \tau \leq t, \quad u_{\tau} \in L^{2}(\Omega) .
$$

Firstly, we establish the following natural result, the multi-valued map $U^{\varepsilon}$ is a strict multi-valued process. Roughly speaking, this is a consequence of the translation and concatenation properties of the weak solutions. The proof is not difficult, so we omit it for the sake of brevity.

Lemma 1 Assume that the function $a \in C\left(\mathbb{R} ; \mathbb{R}_{+}\right)$fulfils (3), $\varepsilon \in[0,1), f \in$ $C(\mathbb{R})$ satisfies (4), $h \in L_{\text {loc }}^{2}\left(\mathbb{R} ; H^{-1}(\Omega)\right)$, and $l \in L^{2}(\Omega)$. Then, the multivalued map $U^{\varepsilon}$ given by $(16)$ is a strict multi-valued process on $L^{2}(\Omega)$ for all $\varepsilon \in[0,1)$.

Remark 2 Observe that when $\varepsilon=0, U^{0}(t, \tau)=\mathcal{S}(t-\tau)$ for all $(t, \tau) \in \mathbb{R}_{d}^{2}$, where $\mathcal{S}$ is the multi-valued semiflow associated to the weak solutions of $\left(P_{0}\right)$. It is obvious that the dynamical system generated by $\left(P_{0}\right)$ is autonomous. We will also keep the notation $\Phi^{0}$ for the set of solutions to $\left(P_{0}\right)$.

Now, for each fixed $\varepsilon$, we have the following continuity result concerning the weak solutions to $\left(P_{\varepsilon}\right)$. To do this, we will apply an energy method which relies on the continuity of weak solutions (cf. [34,35,44,46,33]).

Proposition 1 Under the assumptions of Lemma 1, if $\left\{u_{\tau}^{n}\right\} \subset L^{2}(\Omega)$ is a sequence such that $u_{\tau}^{n} \rightarrow u_{\tau}$ strongly in $L^{2}(\Omega)$, then for any sequence $\left\{u^{n}\right\}$ with $u^{n} \in \Phi^{\varepsilon}\left(\tau, u_{\tau}^{n}\right)$ for each $n \geq 1$, there exist a subsequence of $\left\{u^{n}\right\}$ (relabeled the same) and $u \in \Phi^{\varepsilon}\left(\tau, u_{\tau}\right)$ such that

$$
u^{n}(s) \rightarrow u(s) \quad \text { strongly in } L^{2}(\Omega) \quad \forall s \geq \tau .
$$

Proof Fix $\tau<T$. From the energy equality and taking into account (3), it follows

$$
\frac{1}{2} \frac{d}{d t}\left|u^{n}(t)\right|^{2}+(1-\varepsilon) m\left\|u^{n}(t)\right\|^{2} \leq\left(f\left(u^{n}(t)\right), u^{n}(t)\right)+\varepsilon\left\langle h(t), u^{n}(t)\right\rangle
$$

a.e. $t \in(\tau, T)$. Then, taking into account that

$$
\begin{aligned}
& \left(f\left(u^{n}(t)\right), u^{n}(t)\right) \leq \kappa|\Omega|-\alpha_{2}\left\|u^{n}(t)\right\|_{L^{p}(\Omega)}^{p} \quad \text { a.e. } t>\tau, \\
& \quad \varepsilon\left\langle h(t), u^{n}(t)\right\rangle \leq \frac{\varepsilon^{2}\|h(t)\|_{*}^{2}}{2(1-\varepsilon) m}+\frac{(1-\varepsilon) m}{2}\left\|u^{n}(t)\right\|^{2} \quad \text { a.e. } t>\tau,
\end{aligned}
$$

it holds

$$
\frac{d}{d t}\left|u^{n}(t)\right|^{2}+(1-\varepsilon) m\left\|u^{n}(t)\right\|^{2}+2 \alpha_{2}\left\|u^{n}(t)\right\|_{L^{p}(\Omega)}^{p} \leq 2 \kappa|\Omega|+\frac{\varepsilon^{2}}{(1-\varepsilon) m}\|h(t)\|_{*}^{2}
$$

a.e. $t>\tau$. Integrating between $\tau$ and $t \in(\tau, T]$, we have

$$
\begin{aligned}
& \left|u^{n}(t)\right|^{2}+(1-\varepsilon) m \int_{\tau}^{t}\left\|u^{n}(s)\right\|^{2} d s+2 \alpha_{2} \int_{\tau}^{t}\left\|u^{n}(s)\right\|_{L^{p}(\Omega)}^{p} d s \\
\leq & \left|u_{\tau}^{n}\right|^{2}+2 \kappa|\Omega|(T-\tau)+\frac{\varepsilon^{2}}{(1-\varepsilon) m} \int_{\tau}^{T}\|h(s)\|_{*}^{2} d s .
\end{aligned}
$$


Thus, the sequence $\left\{u^{n}\right\}$ is bounded in $L^{\infty}\left(\tau, T ; L^{2}(\Omega)\right) \cap L^{2}\left(\tau, T ; H_{0}^{1}(\Omega)\right)$ $\cap L^{p}\left(\tau, T ; L^{p}(\Omega)\right)$. From this, taking into account (3) and (5), we deduce that the sequences $\left\{-a\left(l\left(u^{n}\right)\right) \Delta u^{n}\right\}$ and $\left\{f\left(u^{n}\right)\right\}$ are bounded in $L^{2}\left(\tau, T ; H^{-1}(\Omega)\right)$ and $L^{q}\left(\tau, T ; L^{q}(\Omega)\right)$ respectively. Therefore, the sequence $\left\{\left(u^{n}\right)^{\prime}\right\}$ is bounded in $L^{2}\left(\tau, T ; H^{-1}(\Omega)\right)+L^{q}\left(\tau, T ; L^{q}(\Omega)\right)$. Then, applying the Aubin-Lions lemma, there exists a subsequence of $\left\{u^{n}\right\}$ (relabeled the same) and $u \in L^{\infty}\left(\tau, T ; L^{2}(\Omega)\right.$ ) $\cap L^{2}\left(\tau, T ; H_{0}^{1}(\Omega)\right) \cap L^{p}\left(\tau, T ; L^{p}(\Omega)\right)$ with derivative $u^{\prime} \in L^{2}\left(\tau, T ; H^{-1}(\Omega)\right)+$ $L^{q}\left(\tau, T ; L^{q}(\Omega)\right)$, such that

$$
\left\{\begin{aligned}
& u^{n} \stackrel{*}{\rightarrow} u \quad \text { weakly-star in } L^{\infty}\left(\tau, T ; L^{2}(\Omega)\right), \\
& u^{n} \rightarrow u \quad \text { weakly in } L^{2}\left(\tau, T ; H_{0}^{1}(\Omega)\right), \\
& u^{n} \rightarrow u \quad \text { weakly in } L^{p}\left(\tau, T ; L^{p}(\Omega)\right), \\
& u^{n} \rightarrow u \quad \text { strongly in } L^{2}\left(\tau, T ; L^{2}(\Omega)\right), \\
& u^{n}(s) \rightarrow u(s) \quad \text { strongly in } L^{2}(\Omega), \text { a.e. }(\tau, T), \\
&\left(u^{n}\right)^{\prime} \rightarrow u^{\prime} \quad \text { weakly in } L^{2}\left(\tau, T ; H^{-1}(\Omega)\right)+L^{q}\left(\tau, T ; L^{q}(\Omega)\right), \\
& f\left(u^{n}\right) \rightarrow f(u) \quad \text { weakly in } L^{q}\left(\tau, T ; L^{q}(\Omega)\right), \\
&-a\left(l\left(u^{n}\right)\right) \Delta u^{n} \rightarrow-a(l(u)) \Delta u \quad \text { weakly in } L^{2}\left(\tau, T ; H^{-1}(\Omega)\right),
\end{aligned}\right.
$$

where the limits of the two last convergences have been obtained applying [41, Lemme 1.3, p. 12].

From (18) it is not difficult to check that $u$ fulfils $(6)$ in the interval $(\tau, T)$. In addition, taking into account that $u \in C\left([\tau, T] ; L^{2}(\Omega)\right)$ and arguing as in the proof of Theorem 1 , it holds that $u(\tau)=u_{\tau}$. Therefore, $u \in \Phi^{\varepsilon}\left(\tau, u_{\tau}\right)$.

Now we prove (17). By the boundedness of $\left\{\left(u^{n}\right)^{\prime}\right\}$ in $L^{2}\left(\tau, T ; H^{-1}(\Omega)\right)+$ $L^{q}\left(\tau, T ; L^{q}(\Omega)\right)$, it holds that $\left\{u^{n}\right\}$ is equicontinuous in $H^{-1}(\Omega)+L^{q}(\Omega)$ on $[\tau, T]$. Besides, since $\left\{u^{n}\right\}$ is bounded in $C\left([\tau, t] ; L^{2}(\Omega)\right)$ and the embedding $L^{2}(\Omega) \hookrightarrow H^{-1}(\Omega)+L^{q}(\Omega)$ is compact, applying the Ascoli-Arzelà Theorem, we have (for another sequence, relabeled again the same) that

$$
u^{n} \rightarrow u \quad \text { strongly in } C\left([\tau, T] ; H^{-1}(\Omega)+L^{q}(\Omega)\right) .
$$

From this, and taking into account the boundedness of $\left\{u^{n}\right\}$ in $C\left([\tau, T] ; L^{2}(\Omega)\right)$, we obtain

$$
u^{n}(s) \rightarrow u(s) \quad \text { weakly in } L^{2}(\Omega) \quad \forall s \in[\tau, T] .
$$

On the other hand, the following estimate

$$
|z(s)|^{2} \leq|z(r)|^{2}+2 \kappa|\Omega|(s-r)+\frac{\varepsilon^{2}}{2(1-\varepsilon) m} \int_{r}^{s}\|h(\theta)\|_{*}^{2} d \theta \quad \forall \tau \leq r \leq s \leq T
$$

holds with $z$ replaced by $u$ or any $u^{n}$.

Now, we define the following functions

$$
\begin{gathered}
J_{n}(s)=\left|u^{n}(s)\right|^{2}-2 \kappa|\Omega| s-\frac{\varepsilon^{2}}{2(1-\varepsilon) m} \int_{\tau}^{s}\|h(r)\|_{*}^{2} d r, \\
J(s)=|u(s)|^{2}-2 \kappa|\Omega| s-\frac{\varepsilon^{2}}{2(1-\varepsilon) m} \int_{\tau}^{s}\|h(r)\|_{*}^{2} d r .
\end{gathered}
$$


Observe that the functions $J$ and $J_{n}$ are continuous on $[\tau, T]$ for all $n$ thanks to the regularity of $u$ and all $u^{n}$. In addition, by (20), these functions are non-increasing on $[\tau, T]$. Now, taking into account (18), one deduces that

$$
J_{n}(s) \rightarrow J(s) \quad \text { a.e. } s \in(\tau, T) .
$$

In fact, it can be proved that

$$
J_{n}(s) \rightarrow J(s) \quad \forall s \in[\tau, T] .
$$

To do this, consider fixed $s_{0} \in(\tau, T]$. Now we will prove that $J_{n}\left(s_{0}\right) \rightarrow J\left(s_{0}\right)$.

Let $\left\{s_{m}\right\}_{m \geq 1} \subset(\tau, T)$ be a sequence such that $J_{n}\left(s_{m}\right) \rightarrow J\left(s_{m}\right)$ for all $m \geq 1$ and $s_{m} \uparrow s_{0}$. Then, given $\epsilon>0$, there exist $m(\epsilon) \geq 1$ and $n(\epsilon) \geq 1$ such that

$$
\begin{array}{rlrl}
\left|J\left(s_{m}\right)-J\left(s_{0}\right)\right| & <\frac{\epsilon}{2} \quad \forall m \geq m(\epsilon), \\
\left|J_{n}\left(s_{m(\epsilon)}\right)-J\left(s_{m(\epsilon)}\right)\right|<\frac{\epsilon}{2} \quad \forall n \geq n(\epsilon) .
\end{array}
$$

Now, as $J_{n}$ are non-increasing on $[\tau, T]$,

$$
\begin{aligned}
J_{n}\left(s_{0}\right)-J\left(s_{0}\right) & =J_{n}\left(s_{0}\right)-J_{n}\left(s_{m(\epsilon)}\right)+J_{n}\left(s_{m(\epsilon)}\right)-J\left(s_{m(\epsilon)}\right)+J\left(s_{m(\epsilon)}\right)-J\left(s_{0}\right) \\
& \leq\left|J_{n}\left(s_{m(\epsilon)}\right)-J\left(s_{m(\epsilon)}\right)\right|+\left|J\left(s_{m(\epsilon)}\right)-J\left(s_{0}\right)\right| \\
& <\epsilon \quad \forall n \geq n(\epsilon) .
\end{aligned}
$$

This means that $\limsup _{n}\left|u^{n}\left(s_{0}\right)\right|^{2} \leq\left|u\left(s_{0}\right)\right|^{2}$, but from (19) it holds that $\left|u\left(s_{0}\right)\right|^{2} \leq \liminf _{n}\left|u^{n}\left(s_{0}\right)\right|^{2}$ too. Therefore, (21) holds, and thus, from (19) once more, $(17)$ is satisfied in $[\tau, T]$. It is clear that repeating this procedure in $[\tau, T+1],[\tau, T+2]$, and so on, and using a diagonal argument, then (17) holds for all $s \geq \tau$ for a suitable subsequence.

The next result shows that the multi-valued process $U^{\varepsilon}$ is upper-semicontinuous with closed values for all $\varepsilon \in[0,1)$.

Proposition 2 Under the assumptions of Lemma 1, the multi-valued process $U^{\varepsilon}$ is upper semicontinuous with closed values for all $\varepsilon \in[0,1)$.

Proof Consider fixed $\varepsilon \in[0,1)$. To prove that the multi-valued process $U^{\varepsilon}$ is upper-semicontinuous, we argue by contradiction. Assume that there exist $t \geq \tau, u_{\tau} \in L^{2}(\Omega)$, a neighborhood $\mathcal{N}$ of $U^{\varepsilon}(t, \tau) u_{\tau}$, and a sequence $\left\{y_{n}\right\}$ such that $y_{n} \in U^{\varepsilon}(t, \tau) u_{\tau}^{n}$, where $u_{\tau}^{n} \rightarrow u_{\tau}$ in $L^{2}(\Omega)$, and $y_{n} \notin \mathcal{N}$ for all $n \in \mathbb{N}$.

Now, since $y_{n} \in U^{\varepsilon}(t, \tau) u_{\tau}^{n}$, there exists $u^{n} \in S\left(\tau, u_{\tau}^{n}\right)$ such that $u^{n}(t)=y_{n}$.

Taking into account that $u_{\tau}^{n} \rightarrow u_{\tau}$ in $L^{2}(\Omega)$ and using Proposition 1, there exist a subsequence of $\left\{u^{n}\right\}$ (relabeled the same) and $u \in \Phi^{\varepsilon}\left(\tau, u_{\tau}\right)$ such that (17) holds. Therefore, there exists a subsequence of $\left\{y_{n}\right\}$ (relabeled the same) such that $y_{n} \rightarrow u(t)$ strongly in $L^{2}(\Omega)$. This is a contradiction, since $u(t) \in U^{\varepsilon}(t, \tau) u_{\tau}$. Thus, it satisfies that the multi-valued process $U^{\varepsilon}$ is upper-semicontinuous.

Finally, that the multi-valued process $U^{\varepsilon}$ has closed values follows immediately from Proposition 1. 
The following result will be very useful to define a suitable universe in $\mathcal{P}\left(L^{2}(\Omega)\right)$ for our goals.

Proposition 3 Under the assumptions of Lemma 1, consider $u_{\tau} \in L^{2}(\Omega)$. Then, for any $\mu_{\varepsilon} \in\left(0,2(1-\varepsilon) \lambda_{1} m\right)$, a solution $u$ to $\left(P_{\varepsilon}\right)$ fulfils

$$
|u(t)|^{2} \leq e^{-\mu_{\varepsilon}(t-\tau)}\left|u_{\tau}\right|^{2}+\frac{2 \kappa|\Omega|}{\mu_{\varepsilon}}+\frac{\varepsilon^{2} e^{-\mu_{\varepsilon} t}}{2(1-\varepsilon) m-\lambda_{1}^{-1} \mu_{\varepsilon}} \int_{\tau}^{t} e^{\mu_{\varepsilon} s}\|h(s)\|_{*}^{2} d s \forall t \geq \tau .
$$

Proof From the energy equality, using (3) and (4), we deduce

$$
\frac{d}{d t}|u(t)|^{2}+2(1-\varepsilon) m\|u(t)\|^{2} \leq 2 \kappa|\Omega|+2 \varepsilon\|h(t)\|_{*}\|u(t)\| \quad \text { a.e. } t>\tau .
$$

Adding $\pm \mu_{\varepsilon}|u(t)|^{2}$, multiplying by $e^{\mu_{\varepsilon} t}$, and using the Cauchy inequality, we have

$$
\frac{d}{d t}\left(e^{\mu_{\varepsilon} t}|u(t)|^{2}\right) \leq 2 \kappa|\Omega| e^{\mu_{\varepsilon} t}+\frac{\varepsilon^{2} e^{\mu_{\varepsilon} t}}{2(1-\varepsilon) m-\mu_{\varepsilon} \lambda_{1}^{-1}}\|h(t)\|_{*}^{2} \quad \text { a.e. } t>\tau .
$$

Finally, integrating between $\tau$ and $t,(22)$ holds.

Now, we can define a suitable tempered universe in $\mathcal{P}\left(L^{2}(\Omega)\right)$.

Definition 9 The class of all families of nonempty subsets $\widehat{D}=\{D(t): t \in$ $\mathbb{R}\} \subset \mathcal{P}\left(L^{2}(\Omega)\right)$ such that

$$
\lim _{\tau \rightarrow-\infty}\left(e^{\mu \tau} \sup _{v \in D(\tau)}|v|^{2}\right)=0
$$

is denoted by $\mathcal{D}_{\mu}^{L^{2}}$ for all $\mu>0$.

It is worth noting that $\mathcal{D}_{F}^{L^{2}} \subset \mathcal{D}_{\mu}^{L^{2}}$ and $\mathcal{D}_{\mu}^{L^{2}}$ is inclusion-closed.

To prove the existence of a pullback absorbing family, we will assume that there exist a couple of values $\varepsilon_{0} \in(0,1)$ and $\mu_{\varepsilon_{0}} \in\left(0,2\left(1-\varepsilon_{0}\right) \lambda_{1} m\right)$ such that the function $h$ satisfies

$$
\int_{-\infty}^{0} e^{\mu_{\varepsilon_{0}} s}\|h(s)\|_{*}^{2} d s<\infty .
$$

Actually, once that such couple $\left(\varepsilon_{0}, \mu_{\varepsilon_{0}}\right)$ does exist, it holds trivially that for any $\varepsilon \in\left[0, \varepsilon_{0}\right)$ it is possible to obtain the above estimate for some $\mu_{\varepsilon} \in$ $\left(0,2(1-\varepsilon) \lambda_{1} m\right)$. Namely, it suffices to use $\mu_{\varepsilon}=\mu_{\varepsilon_{0}}$.

Proposition 4 Under the assumptions of Lemma 1, if the function $h$ also satisfies (23), then, for any $\varepsilon \in\left[0, \varepsilon_{0}\right]$ the family $\widehat{D}_{0}^{\varepsilon}=\left\{D_{0}^{\varepsilon}(t): t \in \mathbb{R}\right\}$ defined by $D_{0}^{\varepsilon}(t)=\bar{B}_{L^{2}}\left(0,\left(R_{L^{2}}^{\varepsilon}(t)\right)^{1 / 2}\right)$, the closed ball in $L^{2}(\Omega)$ of center zero and radius $\left(R_{L^{2}}^{\varepsilon}(t)\right)^{1 / 2}$, where

$$
R_{L^{2}}^{\varepsilon}(t)=1+\frac{2 \kappa|\Omega|}{\mu_{\varepsilon}}+\frac{\varepsilon^{2} e^{-\mu_{\varepsilon} t}}{2(1-\varepsilon) m-\lambda_{1}^{-1} \mu_{\varepsilon}} \int_{-\infty}^{t} e^{\mu_{\varepsilon} s}\|h(s)\|_{*}^{2} d s,
$$


is pullback $\mathcal{D}_{\mu_{\varepsilon}}^{L^{2}}$-absorbing for the multi-valued process $U^{\varepsilon}: \mathbb{R}_{d}^{2} \times L^{2}(\Omega) \rightarrow$ $L^{2}(\Omega)$. Besides, $\widehat{D}_{0}^{\varepsilon} \in \mathcal{D}_{\mu_{\varepsilon}}^{L^{2}}$.

Proof Consider fixed $\varepsilon \in\left[0, \varepsilon_{0}\right]$ and $u_{\tau} \in D(\tau) \in \widehat{D} \in \mathcal{D}_{\mu_{\varepsilon}}^{L^{2}}$. Given $(t, \tau) \in \mathbb{R}_{d}^{2}$ and $u \in \Phi^{\varepsilon}\left(\tau, u_{\tau}\right)$, from Proposition 3, we know that $u$ fulfils (22). Now, since $\widehat{D} \in \mathcal{D}_{\mu_{\varepsilon}}^{L^{2}}$, there exists $\tau_{0}(\widehat{D}, t)<t$ such that

$$
e^{-\mu_{\varepsilon}(t-\tau)}\left|u_{\tau}\right|^{2} \leq 1 \quad \forall \tau \leq \tau_{0}(\widehat{D}, t) .
$$

Therefore, from this, (22), and the fact that $h$ satisfies (23), we conclude the result.

Now, to prove the pullback $\mathcal{D}_{\mu_{\varepsilon}}^{L^{2}}$-asymptotic compactness, we will use the following estimates.

Lemma 2 Under the assumptions of Proposition 4, for any $\varepsilon \in\left[0, \varepsilon_{0}\right], t \in \mathbb{R}$, and $\widehat{D} \in \mathcal{D}_{\mu_{\varepsilon}}^{L^{2}}$, there exists $\tau_{1}(\widehat{D}, t)<t-2$, such that for any $\tau \leq \tau_{1}(\widehat{D}, t)$ and any $u_{\tau} \in D(\tau)$, the solutions to $\left(P_{\varepsilon}\right)$ satisfy

$$
\left\{\begin{aligned}
\left|u\left(r ; \tau, u_{\tau}\right)\right|^{2} & \leq \rho_{1}^{\varepsilon}(t) \quad \forall r \in[t-2, t], \\
\int_{r-1}^{r}\left\|u\left(s ; \tau, u_{\tau}\right)\right\|^{2} d s & \leq \rho_{2}^{\varepsilon}(t) \quad \forall r \in[t-1, t], \\
\int_{r-1}^{r}\left\|u\left(s ; \tau, u_{\tau}\right)\right\|_{L^{p}(\Omega)}^{p} d s & \leq \frac{(1-\varepsilon) m}{2 \alpha_{2}} \rho_{2}^{\varepsilon}(t) \quad \forall r \in[t-1, t],
\end{aligned}\right.
$$

where

$$
\begin{aligned}
& \rho_{1}^{\varepsilon}(t)=1+\frac{2 \kappa|\Omega|}{\mu_{\varepsilon}}+\frac{\varepsilon^{2} e^{-\mu_{\varepsilon}(t-2)}}{2(1-\varepsilon) m-\lambda_{1}^{-1} \mu_{\varepsilon}} \int_{-\infty}^{t} e^{\mu_{\varepsilon} s}\|h(s)\|_{*}^{2} d s, \\
& \rho_{2}^{\varepsilon}(t)=\frac{1}{(1-\varepsilon) m}\left(\rho_{1}^{\varepsilon}(t)+2 \kappa|\Omega|+\frac{\varepsilon^{2}}{(1-\varepsilon) m} \max _{r \in[t-1, t]} \int_{r-1}^{r}\|h(s)\|_{*}^{2} d s\right) .
\end{aligned}
$$

Proof The first inequality of (24) involving the expression $\rho_{1}^{\varepsilon}$ can be proved analogously as in the proof of Proposition 4 if $\tau \leq \tau_{1}(\widehat{D}, t)<t-2$ (far enough pullback in time) because of our choice of tempered universe and taking into account (23). As in Proposition 1, we have

$$
\frac{1}{2} \frac{d}{d s}|u(s)|^{2}+(1-\varepsilon) m\|u(s)\|^{2} \leq(f(u(s)), u(s))+\varepsilon\langle h(s), u(s)\rangle \quad \text { a.e. } s>\tau .
$$

Now, using (4) and the Cauchy inequality, we have

$$
\frac{d}{d s}|u(s)|^{2}+(1-\varepsilon) m\|u(s)\|^{2}+2 \alpha_{2}\|u(s)\|_{L^{p}(\Omega)}^{p} \leq 2 \kappa|\Omega|+\frac{\varepsilon^{2}}{(1-\varepsilon) m}\|h(s)\|_{*}^{2}
$$


a.e. $s>\tau$. Then, we deduce for all $\tau \leq r-1$ that

$$
\begin{aligned}
& |u(r)|^{2}+(1-\varepsilon) m \int_{r-1}^{r}\|u(s)\|^{2} d s+2 \alpha_{2} \int_{r-1}^{r}\|u(s)\|_{L^{p}(\Omega)}^{p} d s \\
\leq & |u(r-1)|^{2}+2 \kappa|\Omega|+\frac{\varepsilon^{2}}{(1-\varepsilon) m} \int_{r-1}^{r}\|h(s)\|_{*}^{2} d s .
\end{aligned}
$$

In particular, from above and the first inequality in (24), we conclude the proof.

Now, we are ready to prove that for any $\varepsilon$ small enough (namely, less than or equal to $\varepsilon_{0}$, after $(23)$ ), the multi-valued process $U^{\varepsilon}$ is pullback $\mathcal{D}_{\mu_{\varepsilon}}^{L^{2}}$ asymptotically compact. To that end, we argue analogously as in the proof of Proposition 1, making use of continuous and non-increasing functions. We only provide a brief sketch and omit the details, for the sake of brevity.

Proposition 5 Under the assumptions of Proposition 4, for any $\varepsilon \in\left[0, \varepsilon_{0}\right]$, the multi-valued process $U^{\varepsilon}$ is pullback $\mathcal{D}_{\mu_{\varepsilon}}^{L^{2}}$-asymptotically compact.

Proof Fix $\varepsilon \in\left[0, \varepsilon_{0}\right], t \in \mathbb{R}$, a family $\widehat{D} \in \mathcal{D}_{\mu_{\varepsilon}}^{L^{2}}$, a sequence $\left\{\tau_{n}\right\} \subset(-\infty, t-2]$ with $\tau_{n} \rightarrow-\infty$, and $u_{\tau_{n}} \in D\left(\tau_{n}\right)$ for all $n$. Let us prove that any sequence $\left\{y^{n}\right\}$, where $y^{n} \in U^{\varepsilon}\left(t, \tau_{n}\right) u_{\tau_{n}}$ for all $n$, is relatively compact in $L^{2}(\Omega)$. Observe that since $y^{n} \in U^{\varepsilon}\left(t, \tau_{n}\right) u_{\tau_{n}}$, there exists $u^{n} \in \Phi^{\varepsilon}\left(\tau_{n}, u_{\tau_{n}}\right)$ such that $u^{n}(t)=$ $y^{n}$.

On account of Lemma 2, there exists $\tau_{1}(\widehat{D}, t)<t-2$ satisfying that, if $n_{1} \geq 1$ is such that $\tau_{n} \leq \tau_{1}(\widehat{D}, t)$ for all $n \geq n_{1},\left\{u^{n}\right\}_{n \geq n_{1}}$ is bounded in $L^{\infty}\left(t-2, t ; L^{2}(\Omega)\right) \cap L^{2}\left(t-2, t ; H_{0}^{1}(\Omega)\right) \cap L^{p}\left(t-2, t ; L^{p}(\Omega)\right)$. With regard to the nonlinear terms, using (3), it holds that $\left\{-a\left(l\left(u^{n}\right)\right) \Delta u^{n}\right\}_{n \geq n_{1}}$ is bounded in $L^{2}\left(t-2, t ; H^{-1}(\Omega)\right)$, and using (5), we can prove that $\left\{f\left(u^{n}\right)\right\}_{n>n_{1}}$ is bounded in $L^{q}\left(t-2, t ; L^{q}(\Omega)\right)$. As a result, $\left\{\left(u^{n}\right)^{\prime}\right\}_{n \geq n_{1}}$ is bounded in $L^{2}(t-$ $\left.2, t ; H^{-1}(\Omega)\right)+L^{q}\left(t-2, t ; L^{q}(\Omega)\right)$. Then, using the Aubin-Lions compactness Lemma, there exists an element $u \in L^{\infty}\left(t-2, t ; L^{2}(\Omega)\right) \cap L^{2}\left(t-2, t ; H_{0}^{1}(\Omega)\right) \cap$ $L^{p}\left(t-2, t ; L^{p}(\Omega)\right)$ with $u^{\prime} \in L^{2}\left(t-2, t ; H^{-1}(\Omega)\right)+L^{q}\left(t-2, t ; L^{q}(\Omega)\right)$, such that for a subsequence (relabeled the same) it follows

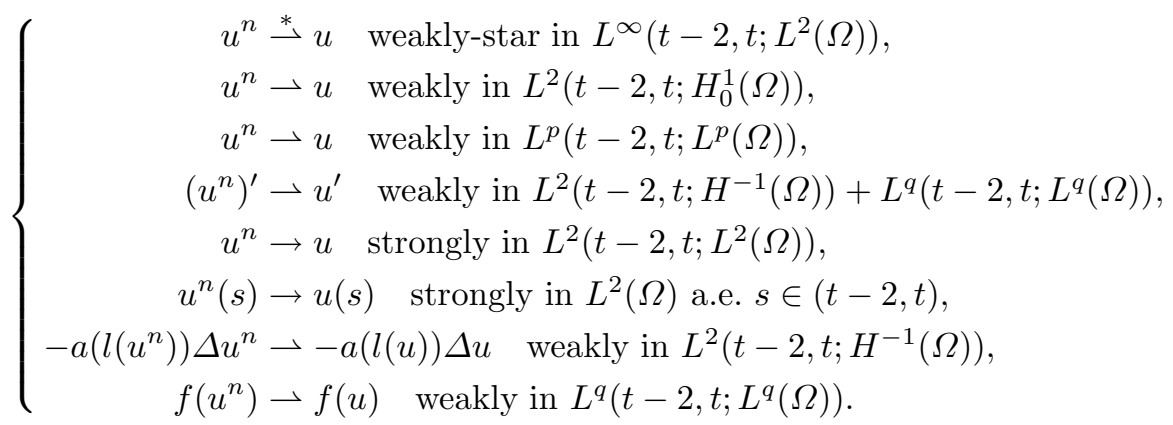

Observe that $u \in C\left([t-2, t] ; L^{2}(\Omega)\right)$, and due to (25), $u$ fulfils (6) in the interval $(t-2, t)$. 
From (25) it holds that $\left\{u^{n}\right\}_{n \geq n_{1}}$ is equicontinuous in $H^{-1}(\Omega)+L^{q}(\Omega)$ on $[t-2, t]$. Moreover, since $\left\{u^{n}\right\}_{n \geq n_{1}}$ is bounded in $C\left([t-2, t] ; L^{2}(\Omega)\right)$ and the embedding $L^{2}(\Omega) \hookrightarrow H^{-1}(\Omega)+L^{q}(\Omega)$ is compact, by the Ascoli-Arzelà Theorem, we have (for another sequence, relabeled again the same) that

$$
u^{n} \rightarrow u \quad \text { strongly in } C\left([t-2, t] ; H^{-1}(\Omega)+L^{q}(\Omega)\right) .
$$

Using the boundedness of $\left\{u^{n}\right\}_{n \geq n_{1}}$ in $C\left([t-2, t] ; L^{2}(\Omega)\right)$, for any sequence $\left\{t_{n}\right\} \subset[t-2, t]$ with $t_{n} \rightarrow t_{*}$ it fulfils

$$
u^{n}\left(t_{n}\right) \rightarrow u\left(t_{*}\right) \quad \text { weakly in } L^{2}(\Omega),
$$

where the weak limit has been identified using (26).

Now, analogously to the proof of Proposition 1, it is not difficult to conclude that

$$
u^{n} \rightarrow u \quad \text { strongly in } C\left([t-1, t] ; L^{2}(\Omega)\right),
$$

which implies in particular that the multi-valued process $U$ is pullback $\mathcal{D}_{\mu_{\varepsilon}}^{L^{2}}$ asymptotically compact. We omit the details for the sake of brevity.

Now, we can establish the main result of this section.

Theorem 3 Assume that the function $a \in C\left(\mathbb{R} ; \mathbb{R}_{+}\right)$satisfies (3), $f \in C(\mathbb{R})$ fulfils (4), $h \in L_{\text {loc }}^{2}\left(\mathbb{R} ; H^{-1}(\Omega)\right)$ and there exist $\varepsilon_{0} \in(0,1)$ and $\mu_{\varepsilon_{0}} \in(0,2(1-$ $\left.\left.\varepsilon_{0}\right) \lambda_{1} m\right)$ such that (23) holds, and $l \in L^{2}(\Omega)$. Then, for all processes $U^{\varepsilon}$ with $\varepsilon \in\left(0, \varepsilon_{0}\right]$, there exist the minimal pullback $\mathcal{D}_{F}^{L^{2}}$-attractor $\mathcal{A}_{\mathcal{D}_{F}^{L^{2}}}^{\varepsilon}=\left\{\mathcal{A}_{\mathcal{D}_{F}^{L^{2}}}^{\varepsilon}(t)\right.$ : $t \in \mathbb{R}\}$, and the minimal pullback $\mathcal{D}_{\mu_{\varepsilon}}^{L^{2}}$-attractor $\mathcal{A}_{\mathcal{D}_{\mu_{\varepsilon}}^{L^{2}}}^{\varepsilon}=\left\{\mathcal{A}_{\mathcal{D}_{\mu_{\varepsilon}}^{L^{2}}}^{\varepsilon}(t): t \in \mathbb{R}\right\}$, which is strictly $U^{\varepsilon}$-invariant.

In addition, the family $\mathcal{A}_{\mathcal{D}_{\mu_{\varepsilon}}^{L^{2}}}^{\varepsilon}$ belongs to $\mathcal{D}_{\mu_{\varepsilon}}^{L^{2}}$ and the following relationships hold

$$
\mathcal{A}_{\mathcal{D}_{F}^{L^{2}}}^{\varepsilon}(t) \subset \mathcal{A}_{\mathcal{D}_{\mu_{\varepsilon}}^{L^{2}}}^{\varepsilon}(t) \subset \bar{B}_{L^{2}}\left(0,\left(R_{L^{2}}^{\varepsilon}(t)\right)^{1 / 2}\right) \quad \forall t \in \mathbb{R} \quad \forall \varepsilon \in\left[0, \varepsilon_{0}\right] .
$$

Moreover, if there exists some $\mu_{\widetilde{\varepsilon}_{0}}$ for some $\widetilde{\varepsilon}_{0} \in\left(0, \varepsilon_{0}\right]$ such that $h$ fulfils

$$
\sup _{s \leq 0}\left(e^{-\mu_{\widetilde{\varepsilon}_{0}} s} \int_{-\infty}^{s} e^{\mu_{\widetilde{\varepsilon}_{0}} \theta}\|h(\theta)\|_{*}^{2} d \theta\right)<\infty
$$

then $\mathcal{A}_{\mathcal{D}_{F}^{L^{2}}}^{\varepsilon}(t)=\mathcal{A}_{\mathcal{D}_{\mu_{\varepsilon}}^{L^{2}}}^{\varepsilon}(t)$ for all $t \in \mathbb{R}$ and for all $\varepsilon \in\left(0, \widetilde{\varepsilon}_{0}\right]$.

Proof Thanks to Corollary 1, the existence of the minimal pullback $\mathcal{D}_{\mu_{\varepsilon}}^{L^{2}}$ attractor $\mathcal{A}_{\mathcal{D}_{\mu_{\varepsilon}}^{L^{2}}}$ and the minimal pullback $\mathcal{D}_{F}^{L^{2}}$-attractor $\mathcal{A}_{\mathcal{D}_{F}^{L^{2}}}$, and the relationship between them are guaranteed. Namely, the upper semicontinuity of the multi-valued process with closed values (cf. Proposition 2), the relationship between the universes, the existence of a pullback $\mathcal{D}_{\mu_{\varepsilon}}^{L^{2}}$-absorbing family (cf. Proposition 4), and the pullback $\mathcal{D}_{\mu_{\varepsilon}}^{L^{2}}$-asymptotic compactness in the $L^{2}$-norm (cf. Proposition 5) hold. 
Besides this, the fact that $\mathcal{A}_{\mathcal{D}_{\mu_{\varepsilon}}^{L^{2}}}(t) \subset{\overline{D_{0}(t)}}^{L^{2}}$ for all $t \in \mathbb{R}$ holds by using Theorem 2. In addition, since $\widehat{D}_{0} \in \mathcal{D}_{\mu_{\varepsilon}}^{L^{2}}$, the set $D_{0}(t)$ is closed for all $t \in$ $\mathbb{R}$, and the universe $\mathcal{D}_{\mu_{\varepsilon}}^{L^{2}}$ is inclusion-closed, the family $\mathcal{A}_{\mathcal{D}_{\mu_{\varepsilon}}^{L^{2}}}$ belongs to the tempered universe $\mathcal{D}_{\mu_{\varepsilon}}^{L^{2}}$. Then, from this, and taking into account that $U^{\varepsilon}$ is a strict multi-valued process, we deduce that the family $\mathcal{A}_{\mathcal{D}_{\mu_{\varepsilon}}^{L^{2}}}$ is in fact the strict minimal pullback $\mathcal{D}_{\mu_{\varepsilon}}^{L^{2}}$ for the multi-valued process $U^{\varepsilon}$.

Finally, taking into account (28), it can be proved that for each $\varepsilon \in\left(0, \widetilde{\varepsilon}_{0}\right)$, $\cup_{t \leq T} R_{L^{2}}^{\varepsilon}(t)$ is bounded for each $T \in \mathbb{R}$, where $R_{L^{2}}^{\varepsilon}$ is given in Proposition 4 . Therefore, for each $U_{\varepsilon \in\left(0, \widetilde{\varepsilon}_{0}\right)}$ both families of attractors coincide (cf. Corollary $2)$.

Remark 3 The case $\varepsilon=0$ can be deduced from the above results, but treated more easily since there is no $h$ term in problem $\left(P_{0}\right)$. Consequently, as said in Remark 2, the equation is autonomous and the generated family of multivalued maps by the solution operator is a multi-valued semiflow $\mathcal{S}$ and satisfies $\mathcal{S}(t-\tau)=U^{0}(t, \tau)$. Under the above assumptions, a global compact attractor $\mathcal{A}^{0}$ in $L^{2}(\Omega)$ exists, and it can be seen as pullback attractor not only for the universe $D_{F}^{L^{2}}$ but also for the tempered universe $D_{\mu_{0}}^{L^{2}}$ where $\mu_{0}=2 \lambda_{1} m$ (cf. Propositions 3 and 4 ). Namely, $\mathcal{A}_{\mathcal{D}_{\mu_{0}}^{L^{2}}}^{0}(t)=\mathcal{A}^{0}$ for all $t \in \mathbb{R}$.

\section{Robustness of attractors w.r.t. the parameter}

Finally, we will study the upper semicontinuous behaviour of the attractors $\mathcal{A}_{\mathcal{D}_{\mu_{\varepsilon}}^{L^{2}}}^{\varepsilon}(t)$ as $\varepsilon \rightarrow 0$ for all $t \in \mathbb{R}$. Namely, we will prove that this family of attractors converges upper semicontinuously to the global compact attractor $\mathcal{A}^{0}$ of the multivalued semiflow $\mathcal{S}$ (or alternatively the multivalued process $\left.U^{0}\right)$ associated to problem $\left(P_{0}\right)$. To do this, roughly speaking we will argue by contradiction and make use of a sequential continuity result in the spirit of $[5$, Theorem 7]. (However, observe that now the parameter $\varepsilon$ is also changing.)

Theorem 4 (A continuous dependence result) Assume that the function $a \in C\left(\mathbb{R} ; \mathbb{R}_{+}\right)$fulfils (3), $f \in C(\mathbb{R})$ satisfies (4), $h \in L_{\text {loc }}^{2}\left(\mathbb{R} ; H^{-1}(\Omega)\right)$, and $l \in L^{2}(\Omega)$. Consider also sequences $\left\{\varepsilon_{n}\right\} \subset(0,1)$ with $\lim _{n} \varepsilon_{n}=0$ and $\left\{u_{\tau}^{n}\right\} \subset$ $L^{2}(\Omega)$ such that $u_{\tau}^{n} \rightarrow u_{\tau}$ weakly in $L^{2}(\Omega)$. Then, there exist a subsequence of $\left\{u_{\tau}^{n}\right\}$ (relabeled the same), $u^{\varepsilon_{n}} \in \Phi^{\varepsilon_{n}}\left(\tau, u_{\tau}^{n}\right)$, and $u^{0} \in \Phi^{0}\left(\tau, u_{\tau}\right)$ such that for 
all $T>\tau$ the following convergences hold:

$$
\left\{\begin{aligned}
& u^{\varepsilon_{n}} \stackrel{*}{\rightarrow} u^{0} \quad \text { weakly-star in } L^{\infty}\left(\tau, T ; L^{2}(\Omega)\right), u^{\varepsilon_{n}} \rightarrow u^{0} \quad \text { weakly in } L^{2}\left(\tau, T ; H_{0}^{1}(\Omega)\right), \\
& u^{\varepsilon_{n}} \rightarrow u^{0} \quad \text { weakly in } L^{p}\left(\tau, T ; L^{p}(\Omega)\right), \\
& u^{\varepsilon_{n}} \rightarrow u^{0} \quad \text { strongly in } L^{2}\left(\tau, T ; L^{2}(\Omega)\right), \\
& f\left(u^{\varepsilon_{n}}\right) \rightarrow f\left(u^{0}\right) \quad \text { weakly in } L^{q}\left(\tau, T ; L^{q}(\Omega)\right), \\
&-a\left(l\left(u^{\varepsilon_{n}}\right)\right) \Delta u^{\varepsilon_{n}} \rightarrow-a\left(l\left(u^{0}\right)\right) \Delta u^{0} \quad \text { weakly in } L^{2}\left(\tau, T ; H^{-1}(\Omega)\right), \\
&\left(u^{\varepsilon_{n}}\right)^{\prime} \rightarrow\left(u^{0}\right)^{\prime} \quad \text { weakly in } L^{2}\left(\tau, T ; H^{-1}(\Omega)\right)+L^{q}\left(\tau, T ; L^{q}(\Omega)\right), \\
& u^{\varepsilon_{n}}(t) \rightarrow u^{0}(t) \quad \text { strongly in } L^{2}(\Omega) \text { for all } t>\tau .
\end{aligned}\right.
$$

Proof Step 1: We will establish all the convergences in (29) but the last.

Consider fixed $(T, \tau) \in \mathbb{R}_{d}^{2}$ and a sequence $\left\{u_{\tau}^{n}\right\}$ such that $u_{\tau}^{n} \rightarrow u_{\tau}$ weakly in $L^{2}(\Omega)$. We will prove this step 1 in the interval $(\tau, T)$. It is standard, using the same procedure in intervals of the form $(\tau, T+1),(\tau, T+2)$, etcetera, and a diagonal argument, that all the convergences (but the last one) hold for all $T>\tau$.

Since each $u^{\varepsilon_{n}}$ is a weak solution to $\left(\mathrm{P}_{\varepsilon_{n}}\right)$ in $[\tau, T]$, from the energy equality, using (3), we have

$$
\frac{1}{2} \frac{d}{d t}\left|u^{\varepsilon_{n}}(t)\right|^{2}+\left(1-\varepsilon_{n}\right) m\left\|u^{\varepsilon_{n}}(t)\right\|^{2} \leq\left(f\left(u^{\varepsilon_{n}}(t)\right), u^{\varepsilon_{n}}(t)\right)+\varepsilon_{n}\left\langle h(t), u^{\varepsilon_{n}}(t)\right\rangle
$$

a.e. $t \in[\tau, T]$. Observe that $\gamma:=\max _{n}\left\{\varepsilon_{n}\right\} \in(0,1)$. Then, using this, (4) and the Cauchy inequality, we obtain

$$
\frac{d}{d t}\left|u^{\varepsilon_{n}}(t)\right|^{2}+(1-\gamma) m\left\|u^{\varepsilon_{n}}(t)\right\|^{2}+2 \alpha_{2}\left\|u^{\varepsilon_{n}}(t)\right\|_{L^{p}(\Omega)}^{p} \leq 2 \kappa|\Omega|+\frac{\varepsilon_{n}^{2}\|h(t)\|_{*}^{2}}{\left(1-\varepsilon_{n}\right) m}
$$

a.e. $t \in[\tau, T]$. From this, we deduce that $\left\{u^{\varepsilon_{n}}\right\}$ is bounded in $L^{\infty}\left(\tau, T ; L^{2}(\Omega)\right) \cap$ $L^{2}\left(\tau, T ; H_{0}^{1}(\Omega)\right) \cap L^{p}\left(\tau, T ; L^{p}(\Omega)\right)$. Besides, using (3) and taking into account the boundedness of $\left\{u^{\varepsilon_{n}}\right\}$ in $L^{2}\left(\tau, T ; H_{0}^{1}(\Omega)\right)$, we deduce that $\left\{-a\left(l\left(u^{\varepsilon_{n}}\right)\right) \Delta u^{\varepsilon_{n}}\right\}$ is bounded in $L^{2}\left(\tau, T ; H^{-1}(\Omega)\right)$. Finally, from (5) and the boundedness of $\left\{u^{\varepsilon_{n}}\right\}$ in $L^{p}\left(\tau, T ; L^{p}(\Omega)\right)$, we obtain that $\left\{f\left(u^{\varepsilon_{n}}\right)\right\}$ is bounded in $L^{q}\left(\tau, T ; L^{q}(\Omega)\right)$. From all this and bearing in mind that

$\frac{d u^{\varepsilon_{n}}}{d t}=\left(1-\varepsilon_{n}\right) a\left(l\left(u^{\varepsilon_{n}}\right)\right) \Delta u^{\varepsilon_{n}}+f\left(u^{\varepsilon_{n}}\right)+\varepsilon_{n} h \quad$ in $\mathcal{D}^{\prime}\left(\tau, T ; H^{-1}(\Omega)+L^{q}(\Omega)\right)$,

we have that $\left\{\left(u^{\varepsilon_{n}}\right)^{\prime}\right\}$ is bounded in $L^{2}\left(\tau, T ; H^{-1}(\Omega)\right)+L^{q}\left(\tau, T ; L^{q}(\Omega)\right)$.

Then, using the Aubin-Lions lemma, there exist a subsequence of $\left\{u^{\varepsilon_{n}}\right\}$ (relabeled the same), $u^{0} \in L^{2}\left(\tau, T ; H_{0}^{1}(\Omega)\right) \cap L^{p}\left(\tau, T ; L^{p}(\Omega)\right) \cap L^{\infty}\left(\tau, T ; L^{2}(\Omega)\right)$ with $\left(u^{0}\right)^{\prime} \in L^{2}\left(\tau, T ; H^{-1}(\Omega)\right)+L^{q}\left(\tau, T ; L^{q}(\Omega)\right)$, such that (29) holds.

Actually, by the Dominated Convergence Theorem, we will also assume that this subsequence is such that

$$
u^{\varepsilon_{n}}(t) \rightarrow u^{0}(t) \quad \text { strongly in } L^{2}(\Omega) \text { a.e. } t \in(\tau, T) .
$$


Now, we check that $u^{0}(t) \in U^{0}(t, \tau) u_{\tau}$ with $t \in[\tau, T]$. Since $u^{\varepsilon_{n}}$ is a weak solution to $\left(P_{\varepsilon_{n}}\right)$, if one considers $v \in H_{0}^{1}(\Omega) \cap L^{p}(\Omega)$ and $\varphi \in \mathcal{D}(\tau, T)$, then

$$
\begin{aligned}
& -\int_{\tau}^{T}\left(u^{\varepsilon_{n}}(t), v\right) \varphi^{\prime}(t) d t+\left(1-\varepsilon_{n}\right) \int_{\tau}^{T} a\left(l\left(u^{\varepsilon_{n}}(t)\right)\right)\left(\left(u^{\varepsilon_{n}}(t), v\right)\right) \varphi(t) d t \\
= & \int_{\tau}^{T}\left(f\left(u^{\varepsilon_{n}}(t)\right), v\right) \varphi(t) d t+\varepsilon_{n} \int_{\tau}^{T}\langle h(t), v\rangle \varphi(t) d t .
\end{aligned}
$$

Using (29) and taking into account that $\lim _{n \rightarrow \infty} \varepsilon_{n}=0$, then

$$
-\int_{\tau}^{T}\left(u^{0}(t), v\right) \varphi^{\prime}(t) d t+\int_{\tau}^{T} a\left(l\left(u^{0}(t)\right)\right)\left(\left(u^{0}(t), v\right)\right) \varphi(t) d t=\int_{\tau}^{T}\left(f\left(u^{0}(t)\right), v\right) \varphi(t) d t .
$$

Therefore, $u^{0}$ is a solution to the equation in $\left(P_{0}\right)$. To conclude that $u^{0} \in$ $\Phi^{0}\left(\tau, u_{\tau}\right)$, we need to check that $u^{0}(\tau)=u_{\tau}$. But this is standard, using $v \in H_{0}^{1}(\Omega) \cap L^{p}(\Omega)$ and $\varphi \in H^{1}(\tau, T)$, with $\varphi(T)=0$ and $\varphi(\tau) \neq 0$, as test elements in the problems $\left(P_{\varepsilon_{n}}\right)$ in $[\tau, T]$. Then, after passing to the limit, bearing in mind (29) and the fact that $u_{\tau}^{n} \rightarrow u_{\tau}$ weakly in $L^{2}(\Omega)$, we deduce

$$
\begin{aligned}
& -\int_{\tau}^{T}\left(u^{0}(t), v\right) \varphi^{\prime}(t) d t-\left(u_{\tau}, v\right) \varphi(\tau)+\int_{\tau}^{T} a\left(l\left(u^{0}(t)\right)\right)\left(\left(u^{0}(t), v\right)\right) \varphi(t) d t \\
= & \int_{\tau}^{T}\left(f\left(u^{0}(t)\right), v\right) \varphi(t) d t .
\end{aligned}
$$

On the other hand, the same test element $v \varphi$ in $\left(P_{0}\right)$, after integration between $\tau$ and $T$, yields

$$
\begin{aligned}
& -\int_{\tau}^{T}\left(u^{0}(t), v\right) \varphi^{\prime}(t) d t-\left(u^{0}(\tau), v\right) \varphi(\tau)+\int_{\tau}^{T} a\left(l\left(u^{0}(t)\right)\right)\left(\left(u^{0}(t), v\right)\right) \varphi(t) d t \\
= & \int_{\tau}^{T}\left(f\left(u^{0}(t)\right), v\right) \varphi(t) d t .
\end{aligned}
$$

Comparing both expressions, since $\varphi(\tau) \neq 0$ and $H_{0}^{1}(\Omega) \cap L^{p}(\Omega)$ is dense in $L^{2}(\Omega)$, it turns out that $u^{0}(\tau)=u_{\tau}$.

Step 2: The last convergence in (29) holds.

Observe that from the energy equality (7), we deduce

$$
\left|u^{\varepsilon_{n}}(s)\right|^{2} \leq\left|u^{\varepsilon_{n}}(r)\right|^{2}+2 \kappa|\Omega|(s-r)+\frac{\varepsilon_{n}^{2}}{2\left(1-\varepsilon_{n}\right) m} \int_{r}^{s}\|h(\theta)\|_{*}^{2} d \theta \forall \tau \leq r \leq s \leq T .
$$

Analogously, $u^{0}$ fulfils

$$
\left|u^{0}(s)\right|^{2} \leq\left|u^{0}(r)\right|^{2}+2 \kappa|\Omega|(s-r) \quad \forall \tau \leq r \leq s \leq T .
$$

Then, we have the following continuous functions on $[\tau, T]$

$$
\begin{aligned}
J_{\varepsilon_{n}}(s) & =\left|u^{\varepsilon_{n}}(s)\right|^{2}-2 \kappa|\Omega| s-\frac{\varepsilon_{n}^{2}}{2\left(1-\varepsilon_{n}\right) m} \int_{\tau}^{s}\|h(r)\|_{*}^{2} d r, \\
J_{0}(s) & =\left|u^{0}(s)\right|^{2}-2 \kappa|\Omega| s .
\end{aligned}
$$


From the above inequalities, one deduces that the functions $J_{0}$ and $J_{\varepsilon_{n}}$, for all $n$, are non-increasing on $[\tau, T]$. In addition, from this, (29) and (30), it can be proved analogously as in the proof of Proposition 1 that

$$
J_{\varepsilon_{n}}(s) \rightarrow J_{0}(s) \quad \forall s \in(\tau, T] .
$$

Therefore, we deduce

$$
\lim _{n \rightarrow \infty}\left|u^{\varepsilon_{n}}(s)\right|^{2}=\left|u^{0}(s)\right|^{2} \quad \forall s \in(\tau, T] .
$$

On the other hand, analogously as in the proof of Proposition 5, taking into account the boundedness of $\left\{u^{\varepsilon_{n}}\right\}$ and $\left\{\left(u^{\varepsilon_{n}}\right)^{\prime}\right\}$ in $C\left([\tau, T] ; L^{2}(\Omega)\right)$ and $L^{2}\left(\tau, T ; H^{-1}(\Omega)\right)+L^{q}\left(\tau, T ; L^{q}(\Omega)\right)$ respectively, and the compactness of the embedding $L^{2}(\Omega) \hookrightarrow H^{-1}(\Omega)+L^{q}(\Omega)$, the Ascoli-Arzelà Theorem implies that

$$
u^{\varepsilon_{n}} \rightarrow u^{0} \quad \text { strongly in } C\left([\tau, T] ; H^{-1}(\Omega)+L^{q}(\Omega)\right) .
$$

Since $\left\{u^{\varepsilon_{n}}\right\}$ is bounded in $C\left([\tau, T] ; L^{2}(\Omega)\right)$, we obtain

$$
u^{\varepsilon_{n}}(s) \rightarrow u^{0}(s) \quad \text { weakly in } L^{2}(\Omega) \quad \forall s \in[\tau, T] .
$$

From this, together with (31), we conclude the proof.

Now, we are ready to prove the upper semicontinuous convergence of the attractors $\mathcal{A}_{\mathcal{D}_{\mu_{\varepsilon}}^{L^{2}}}^{\varepsilon}(t)$ to $\mathcal{A}^{0}$ as $\varepsilon \rightarrow 0$ for all $t \in \mathbb{R}$.

Theorem 5 Assume that the function $a \in C\left(\mathbb{R} ; \mathbb{R}_{+}\right)$fulfils (3), $f \in C(\mathbb{R})$ satisfies (4), there exist a couple of values $\varepsilon_{0} \in(0,1)$ and $\mu_{\varepsilon_{0}} \in(0,2(1-$ $\left.\left.\varepsilon_{0}\right) \lambda_{1} m\right)$ such that $h \in L_{l o c}^{2}\left(\mathbb{R} ; H^{-1}(\Omega)\right)$ fufils (23), and $l \in L^{2}(\Omega)$. Then, the family $\left\{\mathcal{A}_{\mathcal{D}_{\mu_{\varepsilon}}^{L^{2}}}^{\varepsilon}(t)\right\}_{\varepsilon \in\left(0, \varepsilon_{0}\right]}$ converges upper semicontinuously as $\varepsilon \rightarrow 0$ to $\mathcal{A}^{0}$, the global compact attractor of $\mathcal{S}$, the multivalued semiflow associated to problem $\left(P_{0}\right)$, i.e.

$$
\lim _{\varepsilon \rightarrow 0} \operatorname{dist}\left(\mathcal{A}_{\mathcal{D}_{\mu_{\varepsilon}}^{L^{2}}}^{\varepsilon}(t), \mathcal{A}^{0}\right)=0 \quad \forall t \in \mathbb{R} .
$$

Proof We establish (32) by contradiction. Assume that there exist $\delta>0$, $t \in \mathbb{R}$, and a sequence $\left\{\varepsilon_{n}\right\}_{n>1} \subset\left(0, \varepsilon_{0}\right]$ with $\lim _{n \rightarrow \infty} \varepsilon_{n}=0$ such that

$$
\operatorname{dist}\left(\mathcal{A}_{\mathcal{D}_{\mu_{\varepsilon_{n}}}^{\varepsilon_{n}}}(t), \mathcal{A}^{0}\right)>\delta \quad \forall n \in \mathbb{N} .
$$

By the negatively invariance of the pullback attractors, solutions to problems $\left(P_{\varepsilon_{n}}\right)$ can be constructed inside the attractors, and from above we deduce that there exists a sequence of such solutions $\left\{u^{\varepsilon_{n}}\right\}_{n \geq 1}$ with $u^{\varepsilon_{n}}(t) \in \mathcal{A}_{\mathcal{D}_{\mu_{\varepsilon_{n}}}^{L^{2}}}(t)$ such that

$$
d\left(u^{\varepsilon_{n}}(t), \mathcal{A}^{0}\right)>\delta \quad \forall n \in \mathbb{N} .
$$

Since $\mathcal{A}_{\mathcal{D}_{\mu_{\varepsilon_{n}}}^{L^{2}}}(t) \subset D_{0}^{\varepsilon_{n}}(t)$ for all $n \in \mathbb{N}$ and $t \in \mathbb{R}$ (cf. Theorem 3), and $D_{0}^{\varepsilon_{n}}(t) \subset D_{0}^{\varepsilon_{0}}(t)$ for all $n \in \mathbb{N}$, we obtain that

$$
\mathcal{A}_{\mathcal{D}_{\mu_{\varepsilon_{n}}^{2}}^{L^{2}}}(t) \subset D_{0}^{\varepsilon_{0}}(t) \quad \forall t \in \mathbb{R}, \quad \forall n \geq 1 .
$$


On the other hand, the pullback $\mathcal{D}_{\mu_{\varepsilon_{0}}}^{L^{2}}$-absorbing family $\widehat{D}_{0}^{\varepsilon_{0}}$ belongs to $\mathcal{D}_{2 \lambda_{1} m}^{L^{2}}$ (see Remark 3$)$, so there exists $\tau\left(t, \widehat{D}_{0}^{\varepsilon_{0}}, \delta\right)<t$ such that

$$
\operatorname{dist}\left(U^{0}(t, \tau) D_{0}^{\varepsilon_{0}}(\tau), \mathcal{A}^{0}\right) \leq \frac{\delta}{2} \quad \forall \tau \leq \tau\left(t, \widehat{D}_{0}^{\varepsilon_{0}}, \delta\right)
$$

From the uniform bound at time $\tau\left(t, \widehat{D}_{0}^{\varepsilon_{0}}, \delta\right)$ of all the pullback attractors, $\left\{u^{\varepsilon_{n}}\left(\tau\left(t, \widehat{D}_{0}^{\varepsilon_{0}}, \delta\right)\right)\right\}_{n \geq 1}$ is bounded and possesses a subsequence (relabeled the same) such that $u^{\varepsilon_{n}}\left(\tau\left(t, \widehat{D}_{0}^{\varepsilon_{0}}, \delta\right)\right) \rightarrow u_{\tau}$ weakly in $L^{2}(\Omega)$.

Now, applying Theorem 4, we deduce that there exists $u^{0} \in \Phi^{0}\left(\tau, u_{\tau}\right)$ and a subsequence of $\left\{\varepsilon_{n}\right\}_{n>1}$ (relabeled the same) such that (29) holds in $\left(\tau\left(t, \widehat{D}_{0}^{\varepsilon_{0}}, \delta\right), t\right)$. In particular, from the last convergence in (29) at time $t$, we deduce that there exists $n_{0} \geq 1$ such that

$$
\left|u^{\varepsilon_{n}}(t)-u^{0}(t)\right| \leq \frac{\delta}{2} \quad \forall n \geq n_{0} .
$$

But taking into account (34) and (35), we obtain

$$
\begin{aligned}
d\left(u^{\varepsilon_{n}}(t), \mathcal{A}^{0}\right) & \leq d\left(u^{\varepsilon_{n}}(t), u^{0}(t)\right)+d\left(u^{0}(t), \mathcal{A}^{0}\right) \\
& \leq \frac{\delta}{2}+\frac{\delta}{2}=\delta \quad \forall n \geq n_{0}
\end{aligned}
$$

which is a contradiction with (33).

\section{Conclusions and final remarks}

A family of parameterized nonlocal reaction diffusion equations has been analysed. We do not impose any regularity on the boundary of the domain. Existence of global weak solutions is established. However, the uniqueness is not guaranteed by the conditions on the viscosity coefficient, which lead to a natural multivalued non-autonomous dynamical system framework. The theory of minimal pullback attractors for a suitable tempered universe has been used to obtain a family of parameterized attractors. The time depending forces take values in $H^{-1}$, which involve the use of an energy method in order to prove asymptotic compactness of the processes. Then, the upper semicontinuity behaviour of this family is proved when the parameter goes to zero, connecting the cited attractors with the one of the corresponding autonomous limit problem. It is worth noting that this last result is even new in the case that uniqueness of solution holds.

Although the form of the parameterized perturbation of problems $\left(P_{\varepsilon}\right)$ looks like simple, this performance has been used for the sake of clarity in the presentation. It is not difficult to check that all the results hold with a more general family of equations as

$$
\frac{d u}{d t}-g_{1}(\varepsilon) a(l(u)) \Delta u=\tilde{g}_{1}(\varepsilon) f(u)+g_{0}(\varepsilon) h(t),
$$


where $g_{0}, g_{1}$, and $\tilde{g}_{1}$ are continuous real functions with values, say, in $[0,1]$ and such that $\lim _{\varepsilon \rightarrow 0} g_{0}(\varepsilon)=0, \lim _{\varepsilon \rightarrow 0} g_{1}(\varepsilon)=\lim _{\varepsilon \rightarrow 0} \tilde{g}_{1}(\varepsilon)=1$. Moreover, other generalizations are also possible, as replacing the continuity assumption on $g_{0}, g_{1}$, and $\tilde{g}_{1}$ given above by monotonicity.

\section{Acknowledgments}

We would like to thank the referees for a careful reading and suggestions on a previous version of this manuscript.

This work has been partially supported by FEDER and Ministerio de Economía y Competitividad (Spain) grant MTM2011-22411, and by Junta de Andalucía grant P12-FQM-1492. M.H.-C. is a fellow of Programa de FPI del Ministerio de Economía y Competitividad, reference BES-2012-053398.

\section{Compliance with ethical standards}

Ethical declaration: on behalf of all my co-authors I hereby declare that: The authors state that this research complies with ethical standards. Funding: The three authors have been partially supported by FEDER and Ministerio de Economía y Competitividad (Spain) grant MTM2011-22411, and by Junta de Andalucía (Proyecto de Excelencia P12-FQM-1492). The second author is a fellow of Programa de FPI del Ministerio de Economía y Competitividad, reference BES-2012-053398.

Conflict of Interest: the authors declare that they have no conflict of interest. This research does not involve either human participants or animals.

The three authors have chosen, voluntarily, to participate in this research project.

Signed: M. Herrera-Cobos.

\section{References}

1. Ovono, A.A.: Asymptotic behaviour for a diffusion equation governed by nonlocal interactions. Electron. J. Differential Equations 134, 01-16 (2010)

2. Anguiano, M., Caraballo, T., Real, J., Valero, J.: Pullback attractors for reactiondiffusion equations in some unbounded domain with an $H^{-1}$-valued non-autonomous forcing term and without uniqueness of solutions. Discrete Contin. Dyn. Syst. 14, 307$326(2010)$

3. Anguiano, M.: Attractors for nonlinear and non-autonomous parabolic PDEs in unbounded domains, PhD-thesis, Universidad de Sevilla (2011)

4. Anguiano, M., Caraballo, T., Real, J.: Existence on pullback attractor for reactiondiffusion equation in some unbounded domain with non-autonomous forcing term in $H^{-1}$. Internat. J. Bifur. Chaos Appl. Sci. Engrg. 20, 2645-2656 (2010)

5. Anguiano, M., Marín-Rubio, P., Real, J.: Pullback attractors for non-autonomous reaction-diffusion equations with dynamical boundary conditions. J. Math. Anal. Appl. 383, 608-618 (2011) 
6. Ansari, R., Ramezannezhad, H., Gholami, R.: Nonlocal beam theory for nonlinear vibrations of embedded multiwalled carbon nanotubes in thermal environment. Nonlinear Dynam. 67, 2241-2254 (2012)

7. Arrieta, J.M., Carvalho, A.N., Rodríguez-Bernal, A.: Upper semicontinuity for attractors of parabolic problems with localized large diffusion and nonlinear boundary conditions. J. Differential Equations 168, 33-59 (2000)

8. Bermúdez, A., Seoane, M.L.: Numerical solution of a nonlocal problem arising in plasma physics. Math. Comput. Modelling 27, 45-59 (1998)

9. Caraballo, T., Chueshov, I., Marín-Rubio, P., Real, J.: Existence and asymptotic behaviour for stochastic heat equations with multiplicative noise in materials with memory. Discrete Contin. Dyn. Syst. 18, 253-270 (2007)

10. Caraballo, T., Langa, J.A., Robinson, J.C.: Upper semicontinuity of attractors for small random perturbations of dynamical systems. Comm. Partial Differential Equations 23 1557-1581 (1998)

11. Caraballo, T., Łukaszewicz, G., Real, J.: Pullback attractors for non-autonomous 2DNavier-Stokes equations in some unbounded domains. C. R. Math. Acad. Sci. Paris 342, 263-268 (2006)

12. Caraballo, T., Herrera-Cobos, M., Marín-Rubio, P.: Long-time behaviour of a nonautonomous parabolic equation with nonlocal diffusion and sublinear terms. Nonlinear Anal. 121, 3-18 (2015)

13. Caraballo, T., Kloeden, P.E.: Non-autonomous attractors for integro-differential evolution equations. Discrete Contin. Dyn. Syst. Ser. S 2, 17-36 (2009)

14. Carvalho, A.N., Rodrigues, H.M., Dłotko, T.: Upper semicontinuity of attractors and synchronization. J. Math. Anal. Appl. 220, 13-41 (1998)

15. Carvalho, A.N., Langa, J.A., Robinson, J.C.: Attractors for infinite-dimensional nonautonomous dynamical systems. Springer, New York (2013)

16. Carrillo, J.A.: On a nonlocal elliptic equation with decreasing nonlinearity arising in plasma physics and heat conduction. Nonlinear Anal. 32, 97-115 (1998)

17. Chang, N.H., Chipot, M.: Nonlinear nonlocal evolution problems. Rev. R. Acad. Cienc. Exactas Fís. Nat. Ser. A Mat. RACSAM 97, 423-445 (2003)

18. Chang, N.H., Chipot, M.: On some model diffusion problems with a nonlocal lower order term. Chinese Ann. Math. Ser. B 24, 147-166 (2003)

19. Chang, N.H., Chipot, M.: On some mixed boundary value problems with nonlocal diffusion. Adv. Math. Sci. Appl. 14, 1-24 (2004)

20. Chepyzhov, V.V., Vishik, M.I.: Attractors for Equations of Mathematical Physics. AMS, Providence, RI (2002)

21. Chipot, M.: Elements of Nonlinear Analysis. Birkhäuser Verlag, Basel (2000)

22. Chipot, M., Lovat, B.: Some remarks on nonlocal elliptic and parabolic problems. Nonlinear Anal. 30, 4619-4627 (1997)

23. Chipot, M., Lovat, B.: On the asymptotic behaviour of some nonlocal problems. Positivity 3, 65-81 (1999)

24. Chipot, M., Molinet, L.: Asymptotic behaviour of some nonlocal diffusion problems. Appl. Anal. 80, 273-315 (2001)

25. Chipot, M., Savistka, T.: Nonlocal p-Laplace equations depending on the $L^{p}$ norm of the gradient. Adv. Differential Equations 19, 997-1020 (2014)

26. Chipot, M., Siegwart, M.: On the asymptotic behaviour of some nonlocal mixed boundary value problems. Nonlinear Analysis and applications: to V. Lakshmikantam on his 80th birthday, 431-449, Kluwer Acad. Publ., Dordrecht (2003)

27. Chipot, M., Valente, V., Caffarelli, G.V.: Remarks on a nonlocal problem involving the Dirichlet energy. Rend. Sem. Mat. Univ. Padova 110, 199-220 (2003)

28. Chipot, M., Zheng, S.: Asymptotic behavior of solutions to nonlinear parabolic equations with nonlocal terms. Asymptot. Anal. 45, 301-312 (2005)

29. Corrêa, F.J.S.A., Menezes, S.D.B., Ferreira, J.: On a class of problems involving a nonlocal operator. Appl. Math. Comput. 147, 475-489 (2004)

30. Crauel, H., Debussche, A., Flandoli, F.: Random attractors. J. Dynam. Differential Equations 9, 307-341 (1997)

31. Dautray, R., Lions, J.L.: Analyse Mathématique et Calcul Numérique pour les Sciences et les Techniques. Masson, Paris (1985) 
32. Evans, L.C.: Partial Differential Equations. AMS, Providence, RI, Vol.19 (1998)

33. García-Luengo, J., Marín-Rubio, P., Real, J.: Pullback attractors in $V$ for nonautonomous 2D-Navier-Stokes equations and their tempered behaviour. J. Differential Equations 252, 4333-4356 (2012)

34. Kapustyan, A.V., Melnik, V.S., Valero, J.: Attractors of multivalued dynamical processes generated by phase-field equations. Internat. J. Bifur. Chaos Appl. Sci. Engrg. 13, 1969-1983 (2003)

35. Kapustyan, A.V., Valero, J.: On the connectedness and asymptotic behaviour of solutions of reaction-diffusion systems, J. Math. Anal. Appl. 323, 614-633 (2006)

36. Kapustyan, A.V., Valero, J.: On the Kneser property for the complex Ginzburg-Landau equation and the Lotka-Volterra system with diffusion, J. Math. Anal. Appl. 357, 254$272(2009)$

37. Kiani, K., Wang, Q.: On the interaction of a single-walled carbon nanotube with a moving nanoparticle using nonlocal Rayleigh, Timoshenko, and higher-order beam theories. Eur. J. Mech. A Solids 31, 179-202 (2012)

38. Kloeden, P.E.: Pullback attractors of nonautonomous semidynamical systems, Stoch. Dyn. 3, 101-112 (2003)

39. Lange, H., Perla Menzala, G.: Rates of decay of a nonlocal beam equation. Differential Integral Equations 10, 1075-1092 (1997)

40. Lei, Y., Adhikari, S., Friswell, M.I.: Vibration of nonlocal Kelvin-Voigt viscoelastic damped Timoshenko beams. Internat. J. Engrg. Sci. 66/67, 1-13 (2013)

41. Lions, J.L.: Quelques Méthodes de Résolution des Problèmes aux Limites Non Lineaires. Dunod, Paris (1969)

42. Lovat, B.: Etudes de quelques problèmes paraboliques non locaux, Thèse, Université de Metz (1995)

43. Marín-Rubio, P.: Attractors for parametric delay differential equations without uniqueness and their upper semicontinuous behaviour. Nonlinear Anal. 68, 3166-3174 (2008)

44. Marín-Rubio, P., Planas, G., Real, J.: Asymptotic behaviour of a phase-field model with three coupled equations without uniqueness. J. Differential Equations 246, 4632-4652 (2009)

45. Marín-Rubio, P., Real, J.: On the relation between two different concepts of pullback attractors for non-autonomous dynamical systems. Nonlinear Anal. 71, 3956-3963 (2009)

46. Marín-Rubio, P., Real, J.: Pullback attractors for 2D-Navier-Stokes equations with delays in continuous and sublinear operators. Discrete Contin. Dyn. Syst. 26, 989-1006 (2010)

47. Melnik, V.S., Valero, J.: On attractors of multi-valued semi-flows and differential inclusions. Set-Valued Anal. 6, 83-111 (1998)

48. Robinson, J.C.: Infinite-Dimensional Dynamical Systems. Cambridge University Press, Cambridge (2001)

49. Rosa, R.: The global attractor for the 2D-Navier-Stokes flow os some unbounded domains. Nonlinear Anal. 32, 71-85 (1998)

50. Sell, G., You, Y.: Dynamics of evolutionary equations. Springer-Verlag, New York (2002)

51. Simsen, J., Ferreira, J.: A global attractor for a nonlocal parabolic problem. Nonlinear Stud. 21, 405-416 (2014)

52. Temam, R.: Navier-Stokes Equations and Nonlinear Functional Analysis, 2nd edn. SIAM, Philadelphia, PA (1995)

53. Temam, R.: Infinite-Dimensional Dynamical Systems in Mechanics and Physics, 2nd edn. Springer, New York (1997)

54. Zou, W., Li, F., Liu, M., Lv, B.: Existence of solutions for a nonlocal problem arising in plasma physics. J. Differential Equations 256, 1653-1682 (2014) 